\title{
ON THE LINEARIZATION OF SOME SINGULAR, NONLINEAR ELLIPTIC PROBLEMS AND APPLICATIONS is
}

\author{
Jesús HERNÁNDEZ ${ }^{\mathrm{a}}$, Francisco J. MANCEBO ${ }^{\mathrm{b}}$, José M. VEGA ${ }^{\mathrm{b}, *}$ \\ a Departamento de Matemáticas, Universidad Autónoma de Madrid, 28049 Madrid, Spain \\ ${ }^{\mathrm{b}}$ E.T.S.I. Aeronáuticos, Universidad Politécnica de Madrid, 28040 Madrid, Spain
}

Received 22 December 1999, revised 25 May 2001

ABSTRACT. - This paper deals with the spectrum of a linear, weighted eigenvalue problem associated with a singular, second order, elliptic operator in a bounded domain, with Dirichlet boundary data. In particular, we analyze the existence and uniqueness of principal eigenvalues. As an application, we extend the usual concepts of linearization and Frechet derivability, and the method of sub and supersolutions to some semilinear, singular elliptic problems.

(C) 2002 L'Association Publications de l'Institut Henri Poincaré. Published by Elsevier B.V. All rights reserved

1991 MSC: 35P05; 35J65

RÉSUMÉ. - On étudie le spectre d'un problème à valeurs propres avec poids associé avec un opérateur elliptique singulier d'ordre deux sur un domaine borné avec condition au bord de Dirichlet. En particulier, on considère l'existence et l'unicité des valeurs propres principales. On donne comme application des extensions des notions de linéarisation et différentielle de Fréchet et de la méthode de sous et sursolutions à quelques problèmes elliptiques semilinéaires singuliers. (C) 2002 L'Association Publications de l'Institut Henri Poincaré. Published by Elsevier B.V. All rights reserved

\section{Introduction}

The standard implicit function theorem $[21,15,12]$ and some extensions such as the Lyapunov-Schmidt method [15,12] are powerful tools for the analysis of nonlinear problems. When applicable they provide the complete solution set in a neighborhood of a given solution. That information sometimes leads to global existence and uniqueness results via continuation techniques. Degree theory $[40,12,43]$, on the other hand, directly provides global (but less precise) existence results that can be made more and more

\footnotetext{
This work was supported by DGI under Grants BFM2001-2363 and REN2000-0766 and by the European Network IHP-RTN-002.

* Corresponding author.

E-mail addresses: jesus.hernandez@uam.es (J. Hernández), mancebo@fmetsia.upm.es (F.J. Mancebo), vega@fmetsia.upm.es (J.M. Vega).
} 
precise when particular solutions are known and their index is well defined. Sub and supersolutions methods [41,3,43,37] yield more precise existence results if more information is available. But both the application of the implicit function theorem and the effective calculation of the index rely on the linearization of the nonlinear problem around a particular solution, which is nontrivial when the coefficients are not sufficiently smooth or the nonlinearities are singular. Linearization is also convenient to systematically construct sequences of sub and supersolutions and is quite useful in the analysis of stability properties. The main object of this paper is to provide the appropriate ingredients to directly extend these tools to the analysis of the positive solutions of some second-order, elliptic problems that exhibit a singularity near the boundary. Although our results apply to more general problems ( $f$ depending also on a parameter, $\mathcal{L}$ replaced by a more general nonlinear, elliptic operator), for the sake of clarity we shall derive them for the semilinear problem

$$
\begin{aligned}
& \mathcal{L} u \equiv-\sum_{i, j=1}^{n} a_{i j}(x) \frac{\partial^{2} u}{\partial x_{i} \partial x_{j}}+\sum_{i=1}^{n} b_{i}(x) \frac{\partial u}{\partial x_{i}}=f(x, u) \text { in } \Omega, \\
& u=0 \quad \text { on } \partial \Omega .
\end{aligned}
$$

under the following assumptions, which hold for some $\alpha$ such that $-1<\alpha<1$ :

(H.1) $\Omega \subset \mathbb{R}^{n}$ is a bounded domain, with a $C^{3, \gamma}$ boundary, for some $\gamma>0$ if $n>1$. Note that the distance from $x \in \Omega$ to $\partial \Omega, d(x)$, defines a function $d \in C^{2, \gamma}\left(\bar{\Omega}_{1}\right)$, with $\Omega_{1}=\left\{x \in \Omega: d(x)<\rho_{1}\right\}$ for some $\rho_{1}>0$.

(H.2) The second order part of the operator $-\mathcal{L}$ is uniformly, strongly elliptic in $\Omega$. Also, for all $i, j, k=1, \ldots, n, a_{i j}=a_{j i} \in C^{3}(\Omega) \cap C(\bar{\Omega}), b_{i} \in C^{2}(\Omega)$, and there is a constant $K$ such that $\left|\partial a_{i j} / \partial x_{k}\right|+\left|b_{i}\right|<K\left[1+d(x)^{\alpha}\right]$ and $\left|\partial^{2} a_{i j} / \partial x_{i} \partial x_{j}\right|+\left|\partial b_{i} / \partial x_{j}\right|<K d(x)^{\alpha-1}$ for all $x \in \Omega$. As a consequence, the functions $a_{i j}, x \rightarrow d(x) \partial a_{i j}(x) / \partial x^{k}$ and $x \rightarrow d(x) b_{i}(x)$ are in $C^{0, \delta}(\bar{\Omega})$ whenever $0<\delta<\min \{\alpha+1, \gamma\}$.

(H.3) There is an integer $m>0$ such that $f, \partial^{j} f / \partial u^{j}, \partial^{j} f / \partial u^{j-1} \partial x_{k} \in C(\Omega \times] 0, \infty[)$ for all $k=1, \ldots, n$ and all $j=1, \ldots, m+1$. And if $u: \Omega \rightarrow \mathbb{R}$ is such that $0<k_{1} d(x)<u(x)<k_{2} d(x)$ for all $x \in \Omega$ and some constants $k_{1}$ and $k_{2}$, then $|f(x, u(x))|<K_{0}\left[1+d(x)^{\alpha}\right]$ and $\left|\partial^{j} f(x, u(x)) / \partial u^{j}\right|+\sum_{k=1}^{n} \mid \partial^{j} f(x, u(x)) /$ $\partial u^{j-1} \partial x_{k} \mid<K_{j} d(x)^{\alpha-j}$ for all $x \in \Omega$, all $k=1, \ldots, n$ and all $j=1, \ldots, m+1$, where $K_{j}$ (can depend on $k_{1}$ and $k_{2}$ but) is independent of $u$.

For convenience we are allowing (in (H.2)) the coefficients $b_{i}$ to exhibit an appropriate singularity at the boundary. Also, we are requiring the coefficients of the operator $\mathcal{L}$ to be such that the adjoint operator $\mathcal{L}^{*}$, defined as

$$
\mathcal{L}^{*} u \equiv-\sum \frac{\partial}{\partial x_{i}}\left(a_{i j} \frac{\partial u}{\partial x_{j}}\right)-\sum \frac{\partial}{\partial x_{i}}\left[\left(b_{i}-\sum \frac{\partial a_{i j}}{\partial x_{j}}\right) u\right],
$$

is such that the equation $\mathcal{L}^{*} u=f(x, u)$ also satisfies (H.2)-(H.3); $\mathcal{L}^{*}$ is the formal adjoint of $\mathcal{L}$ with respect to the inner product of $L_{2}(\Omega)$. Also, since all sums apply to the values $1, \ldots, n$ of the involved indexes, the limits 1 and $n$ are omitted hereafter in the symbol $\Sigma$. Note that assumption (H.3) is satisfied by the usual power-law nonlinearities, $f(x, u)=g(x) u^{\alpha_{1}}$, whenever $\alpha_{1}>-1$ and $g \in C^{1}(\bar{\Omega})$; or, more generally, when $g \in$ 
$C^{1}(\Omega)$ and $|g(x)|<K d(x)^{\alpha_{2}}$, for some $K>0$ and some $\alpha_{2}$ such that $\left|\alpha_{1}+\alpha_{2}\right|<1$. Singular elliptic problems of this type were considered, among many others, by Laetsch [33], Cohen and Laetsch [14], Crandall, Rabinowitz and Tartar [18], Brezis and Oswald [11] and Bandle, Pozio and Tesei [6] in bounded domains, and by Spruck [44], Schatzman [42] and Brezis and Kamin [10] in $\mathbb{R}^{n}$. As a by-product of the results in the paper, in Section 4.1 we shall extend the existence (of strictly positive, classical solutions, in $C^{2}(\Omega) \cap C_{0}^{1}(\bar{\Omega})$ ) result in [18] to nonlinearities of indefinite sign, which are of interest in, e.g., population dynamics $[25,26,36]$. Note that if the nonlinearity $f$ is singular at $u=0$ and is negative for $u>0$ and $x \in \Omega^{\prime} \neq \emptyset$, then the nonnegative solutions of (1.1)-(1.2) can exhibit free boundaries between a region $\bar{\Omega}^{\prime \prime} \subset \Omega^{\prime}$ where $u=0$ ('dead core') and the support of $u$ [20]; these solutions will be excluded from the analysis below, where only (strictly) positive solutions will be considered. In Section 4.1 we shall use the method of sub and supersolutions, as in [6], where quite weak, not necessarily (strictly) positive solutions were obtained.

The linearization of (1.1)-(1.2) around a given positive solution $u$ leads us to consider the linear eigenvalue problem

$$
\mathcal{L} U-M(x) U=\lambda U \quad \text { in } \Omega, \quad U=0 \quad \text { on } \partial \Omega,
$$

where $\mathcal{L}$ is as in (1.1) and $M(x)=f_{u}(x, u(x))$. Thus the natural assumption on the coefficient $M$ is

(H.3') $M \in C^{1}(\Omega)$ and, for all $k=1, \ldots, n, d(x)^{2-\alpha}\left|\partial M(x) / \partial x_{k}\right|$ is bounded in $\Omega$.

As a consequence, the function $x \rightarrow d(x)^{2} M(x)$ is in $C^{0, \delta}(\bar{\Omega})$ whenever $0<\delta<$ $\min \{\alpha+1, \gamma\}$, and $d(x)^{1-\alpha} M(x)$ is bounded in $\Omega$.

Note that we are not requiring $M$ to have a constant sign near $\partial \Omega$. In fact, as we shall see in Section 2 (see Lemma 2.1), that sign can be controlled upon a change of variable that affects both the coefficients $b_{i}$ and the coefficient $M$ itself, with the new coefficients still satisfying (H.2) and (H.3'). This result is of independent interest and appears as surprising at first sight because the sign of $M$ near $\partial \Omega$ plays an important role when applying maximum principles. Similar singular eigenvalue problems in divergence form were considered in [8], where generalized Hardy-Sobolev inequalities [13] (see Remark 2.4 below) were used to prove that the eigenfunctions are in $C^{2}(\Omega) \cap H_{0}^{1}(\Omega)$. Here we shall prove that the eigenfunctions are also in $C_{0}^{1, \delta}(\bar{\Omega})$ for all $\delta$ such that $0<\delta<\min \{\alpha+1, \gamma\}$. A stronger $C^{1}(\bar{\Omega})$-regularity (also for the solutions of (1.1)-(1.2) and of some related linear problems) is necessary in order to apply a straightforward generalization (Appendix B) of the Hopf boundary lemma [38]. This and our assumption that $\alpha>-1$ in (H.3)-(H.3') will prevent us from using $L_{p}$ theory [2], Hardy-Sobolev inequalities and imbedding theorems [1], which provide $C^{1}(\bar{\Omega})$-solutions only if $\alpha>$ $-1 / n$ (see Remark 2.3). Instead, we shall use the (integral) reformulation of the various problems through the Green function of the linear problem $\mathcal{L} u=f$ in $\Omega, u=0$ on $\partial \Omega$, and work in $C_{0}^{1}(\bar{\Omega})$ (or in $C_{0}^{1, \delta}(\bar{\Omega})$ when convenient) but, for the sake of clarity, these problems will be written in differential form in the statements of most results. Note that the requirement $\alpha>-1$ in assumptions (H.2)-(H.3), (H.3') and (H.4) is somewhat optimal when seeking $C^{1}(\bar{\Omega})$-regularity, as the simplest counterexamples [8] readily show. 
In fact, in Section 2 we shall consider a slightly more general linear eigenvalue problem, namely

$$
\mathcal{L} U-M(x) U=\lambda N(x) U \quad \text { in } \Omega, \quad U=0 \quad \text { on } \partial \Omega,
$$

where $\mathcal{L}$ and $M$ are as above and

(H.4) $N$ is strictly positive in $\Omega$, and satisfies assumption (H.3').

That extension is made because it leads to no additional price in the analysis and is of interest in the linear stability analysis of the strictly positive steady states of some singular parabolic problems, such as

$$
(\beta /|\beta|) \partial u^{\beta} / \partial t+\mathcal{L} u=f(x, u) \quad \text { in } \Omega, \quad u=0 \quad \text { on } \partial \Omega,
$$

with $\mathcal{L}$ and $f$ as above and $0<|\beta|<1$. That equation includes the so-called porous media equation ([5] and references given there in) as a particular case if $0<\beta<1$, as seen when rewriting it in terms of the new variable $v=u^{\beta}$. The extension is also useful in the analysis of the more standard case $\beta=1$, see Theorem 4.7 below. But in fact we shall also get some results on the existence of principal eigenvalues of (1.5) when $N$ vanishes in a part of $\Omega$, or changes sign in $\Omega$. That extension do involve an additional price, but it is convenient in some applications (e.g., in the analysis of (1.1)-(1.2), with $f(x, u) \equiv N(x) u^{\alpha}$ and $N$ of indefinite sign, as $\left.\alpha \nearrow 1\right)$.

For convenience we shall also consider the adjoint linearized problem

$$
\mathcal{L}^{*} V-M(x) V=\lambda N(x) V \quad \text { in } \Omega, \quad V=0 \quad \text { on } \partial \Omega,
$$

where $\mathcal{L}^{*}$ is as defined in (1.3), and prove that it has the same spectrum as (1.5).

The paper is organized as follows. Section 2 is devoted to the linear eigenvalue problem (1.5), which is first analyzed in the simplest case $N>0$ (Theorem 2.6). For convenience we also characterize the principal eigenvalue (1.5) in terms of a min-max property (Proposition 2.8), first introduced by Donsker and Varadhan [22] to characterize the principal eigenvalue of second-order elliptic operators in general form in bounded, smooth domains, and extended (essentially as a definition) by Berestycki, Nirenberg and Varadhan [7] to general bounded domains. Also, we analyze the existence and uniqueness of the principal eigenvalue of (1.5) when $N$ changes sign in $\Omega$ (Theorem 2.12) and when $N \geqslant 0$ vanishes in a part of $\Omega$ (Theorem 2.10 and Remark 2.11), to extend a well-known result subsequently proven by Manes and Micheletti [35] for self-adjoint operators and by Hess and Kato [29] for general operators, and some recent results by López-Gómez [34] respectively. In Section 3 we first re-write (1.1)-(1.2) in integral form, via a Green operator, and then consider the Fréchet differentiability of the resulting problem with respect to $u$ (Theorem 3.1) and with respect to a parameter, under appropriate, additional regularity assumptions on the dependence of $f$ on the parameter (Corollary 3.2). Finally, several applications are given in Section 4 that deal with the construction of solutions of (1.1)-(1.2) as limits of sequences of sub and/or supersolutions (Section 4.1), with the stability of the solutions of (1.1)-(1.2) as steady states of the associated parabolic problem (Section 4.2), and with bifurcation problems when $f$ is allowed to depend also on a parameter (Section 4.3). 


\section{The spectrum of the linear eigenvalue problem}

The object of this section is to analyze the spectrum of the linear eigenvalue problem (1.5). But before proceeding with the main results we show that the sign of the coefficient $M$ near $\partial \Omega$ can be controlled through a change of variable.

LEMMA 2.1. - Under the assumptions (H.1)-(H.2) and (H.3'), there are two functions $\varphi^{ \pm} \in C^{2, \delta}(\bar{\Omega})$ for all $\delta$ such that $0<\delta<\delta_{0}=\min \{\gamma, \alpha+1\}$, such that

$$
\varphi^{ \pm}>0 \quad \text { in } \bar{\Omega},
$$

and if $U \in X \equiv C^{2}(\Omega) \cap C_{0}^{1, \delta}(\bar{\Omega})$, with $0<\delta<\delta_{0}$, is a solution of (1.5), then the functions $U^{ \pm}=\varphi^{ \pm} U$ are in $X$ and

$$
\begin{gathered}
\mathcal{L}^{ \pm} U^{ \pm} \equiv-\sum a_{i j}(x) \frac{\partial^{2} U^{ \pm}}{\partial x_{i} \partial x_{j}}+\sum b_{i}^{ \pm}(x) \frac{\partial U^{ \pm}}{\partial x_{i}} \\
=M^{ \pm}(x) U^{ \pm}+\lambda N(x) U^{ \pm}, \quad \text { in } \Omega \\
U^{ \pm}=0, \quad \partial U^{ \pm} / \partial v=\partial U / \partial v \quad \text { on } \partial \Omega,
\end{gathered}
$$

where $v$ is the outward unit normal, the coefficients $b_{i}^{ \pm}$and $M^{ \pm}$satisfy assumptions (H.2) and (H.3'), and

$$
\pm M^{ \pm}>0 \text { in a neighborhood of } \partial \Omega .
$$

Proof. - Let $d(x)=d\left(x_{1}, \ldots, x_{n}\right)$ be the distance from $x$ to $\partial \Omega$ and let $\psi \in$ $C^{3}(] 0, \infty[) \cap C^{0}([0, \infty[)$ be a real function such that

$$
\delta \psi(\eta) \geqslant 0 \text { if } \eta \geqslant 0, \quad \delta \psi(\eta)=\eta^{\delta+1} \text { if } 0 \leqslant \eta \leqslant \varepsilon<\rho_{1}, \quad \psi(\eta)=0 \text { if } \eta>\rho_{1},
$$

where $\delta \neq 0$ is such that $-1<\delta<\alpha$, with $\alpha$ and $\rho_{1}$ as in assumptions (H.1), (H.2) and (H. $\left.3^{\prime}\right)$. The strictly positive constant $\varepsilon$ will be selected below.

Now we define the functions $\varphi^{ \pm}$as $\varphi^{ \pm}(x)=\exp [\mp \psi(d(x))]$. If $U \in X$ is a solution of (1.5) then $U^{ \pm}=\varphi^{ \pm} U$ is such that $U^{ \pm} \in X$ and satisfies (2.2) with

$$
\begin{aligned}
b_{i}^{ \pm}= & b_{i} \mp 2 \psi^{\prime}(d(x)) \sum a_{i j} \frac{\partial d}{\partial x_{j}}, \\
M^{ \pm}= & M \mp \psi^{\prime}(d(x)) \sum b_{i} \frac{\partial d}{\partial x_{i}} \\
& +\sum a_{i j}\left[\frac{\partial d}{\partial x_{i}} \frac{\partial d}{\partial x_{j}}\left(\psi^{\prime}(d(x))^{2} \pm \psi^{\prime \prime}(d(x))\right) \pm \frac{\partial^{2} d}{\partial x_{i} \partial x_{j}} \psi^{\prime}(d(x))\right] .
\end{aligned}
$$

But, according to (2.5),

$$
\pm M^{ \pm} d(x)^{1-\delta} \geqslant 0 \quad \text { if } 0<d(x)<\varepsilon
$$

provided that 


$$
\begin{aligned}
& \pm M(x) d(x)^{1-\alpha} d(x)^{\alpha-\delta}-d(x) \sum \frac{b_{i}}{\delta} \frac{\partial d}{\partial x_{i}} \\
& \quad+\sum a_{i j}\left(\left[1-(\delta+1) d(x)^{\delta+1}\right] \frac{\partial d}{\partial x_{i}} \frac{\partial d}{\partial x_{j}}+d(x) \frac{\partial^{2} d}{\partial x_{i} \partial x_{j}}\right)>0,
\end{aligned}
$$

which holds if $0<d(x)<\varepsilon$ and $\varepsilon$ is sufficiently small, because $|M(x)| d(x)^{1-\alpha}$ is bounded in $\Omega$ and the matrix $\left(a_{i j}\right)$ is positive definite in $\Omega$, according to assumptions (H.2) and (H.3 ${ }^{\prime}$ ). Note that $\varepsilon$ is chosen independently of $U$. Also $b_{i}^{ \pm}$and $M^{ \pm}$satisfy assumptions (H.2) and (H.3 $3^{\prime}$ ) respectively and, according to (2.5), $U^{ \pm}$satisfies (2.3). Thus the proof is complete.

Remark 2.2. - The result above implies that the point spectrum of (2.2), with Dirichlet boundary data

$$
U^{ \pm}=0 \quad \text { on } \partial \Omega
$$

is the same as that of (1.5), provided that the eigenfunctions are in $C^{2}(\Omega) \cap C_{0}^{1}(\bar{\Omega})$ (and this is a natural assumption, as we shall see below). Still, (2.1) and (2.3) imply that

$$
\begin{aligned}
& \left.U=0 \quad \text { (resp., } U>0) \quad \text { if and only if } \quad U^{ \pm}=0 \quad \text { (resp., } U^{ \pm}>0\right), \text { in } \Omega ; \\
& \partial U / \partial v=0 \quad(\text { resp., } \partial U / \partial v<0) \quad \text { if and only if } \quad \partial U^{ \pm} / \partial v=0 \\
& \text { (resp., } \left.\partial U^{ \pm} / \partial v<0\right) \text { on } \partial \Omega .
\end{aligned}
$$

The first property implies that $\lambda$ is a principal eigenvalue of (1.5) if and only if it is a principal eigenvalue of (2.2), (2.6). The second property will be quite useful below to apply a Hopf boundary lemma.

In order to analyze the eigenvalue problem (1.5), we first consider the Green operator of the problem

$$
\mathcal{L} u=M(x) v \quad \text { in } \Omega, \quad u=0 \quad \text { on } \partial \Omega,
$$

defined as $u=G_{1}(v)$, with $v \in C_{0}^{0,1}(\bar{\Omega})$. Since the analysis of this problem is somewhat apart from the remaining part of the paper, it is relegated to Appendix $A$ at the end of the paper, and the result is just stated here; but see Remark 2.4 below.

PROPOSITION 2.3. - Let $\Omega, \mathcal{L}$ and $M$ satisfy assumptions (H.1)-(H.2) and (H.3'). If $v \in C_{0}^{0,1}(\bar{\Omega})$ then (2.7) has a unique solution $u \in C^{2}(\Omega) \cap C^{1, \delta}(\bar{\Omega})$ for all $\delta$ such that $0<\delta<\delta_{0}=\min \{\gamma, \alpha+1\}$. And there is a constant $K$, which (can depend on $\delta$ but) is independent of $v$, such that

$$
\|u\|_{C^{1, \delta}(\bar{\Omega})} \leqslant K\|v\|_{C^{0,1}(\bar{\Omega})} .
$$

Proof. - See Appendix A.

Remark 2.4. - If $\alpha>-1 / n$ then we can use $L_{p}$ estimates to show that, under the assumptions in Proposition 2.3, (2.7) possesses a unique solution $u \in W_{p}^{2}(\Omega)$, for all $p>n$ such that $1+\alpha p>0$, and that $\|u\|_{W_{p}^{2}(\Omega)} \leqslant K\|v\|_{C^{0,1}(\bar{\Omega})}$, with $K$ independent of $v$. This result is readily obtained by first replacing (2.7) by

$$
u+G_{0}\left(\sum b_{i} \partial u / \partial x_{i}\right)=G_{0}(M(x) v)
$$


where $G_{0}: L_{p}(\Omega) \rightarrow W_{p}^{2}(\Omega)$ is the Green operator of the problem $-\sum a_{i j} \partial^{2} u / \partial x_{i} \partial x_{j}=$ $f$ in $\Omega, u=0$ on $\partial \Omega$, and then taking into account that

$$
\left\|\sum b_{i} \partial u / \partial x_{i}\right\|_{L_{p}(\Omega)} \leqslant K_{1}\|u\|_{C^{0,1}(\bar{\Omega})}, \quad\|M(x) v\|_{L_{p}(\Omega)} \leqslant K_{2}\|v\|_{C^{0,1}(\bar{\Omega})}
$$

with $K_{1}$ and $K_{2}$ independent of $u$ and $v$ respectively. The first estimate readily follows from assumption (H.2). The second estimate follows from (H.2) and the inequality

$$
|v(x)| \leqslant d(x)\|v\|_{C^{0,1}(\bar{\Omega})} \quad \text { for all } x \in \Omega,
$$

which holds whenever $v \in C_{0}^{0,1}(\bar{\Omega})$, as readily obtained when applying the mean value theorem between $x$ and that point of $\partial \Omega$ where the distance $d(x)$ is reached; the second estimate (2.10) can also be obtained via Hardy-Sobolev inequalities [13], but this requires that $\alpha \geqslant 0$ if $p>n$. Now, when using (2.10), the continuity of $G_{0}$, the fact that $W_{p}^{2}(\Omega)$ is compactly imbedded into $C^{0,1}(\bar{\Omega})$, standard maximum principles and standard Riesz theory on compact, linear operators, the result readily follows. Thus if $\alpha>-1 / n$ we can proceed with $L_{p}$ theory and imbedding theorems to obtain the results below in a simpler way, which unfortunately is not appropriate to obtain $C^{1}$-regularity up to the boundary if $-1<\alpha \leqslant-1 / n$.

The main ingredient to analyze the spectrum of (1.5) when $N>0$ in $\Omega$ is in the following

PROPOSITION 2.5. - Under the assumptions (H.1)-(H.2), (H.3') and (H.4), there is a constant $k_{0}$ such that if $k>k_{0}$ and $v \in C_{0}^{0,1}(\bar{\Omega})$ then the problem

$$
\mathcal{L} u-M(x) u+k N(x) u=N(x) v \quad \text { in } \Omega, \quad u=0 \quad \text { on } \partial \Omega
$$

has a unique solution $u \in C^{2}(\Omega) \cap C^{1, \delta}(\bar{\Omega})$ for all $\left.\delta \in\right] 0, \delta_{0}\left[\right.$, where $\delta_{0}=\min \{\gamma, \alpha+1\}$, and

$$
\|u\|_{C^{1, \delta}(\bar{\Omega})} \leqslant K\|v\|_{C^{0,1}(\bar{\Omega})},
$$

where $K$ (can depend on $k$ and $\delta$ but) is independent of $v$. If, in addition, $v \geqslant 0$ in $\Omega$ and $v$ is not identically zero, then $\partial u / \partial v<0$ on $\partial \Omega$.

Proof. - We first select $k_{0}$ to ensure uniqueness. To this end we rewrite (2.12) as

$$
\mathcal{L}^{-} u^{-}-M^{-}(x) u^{-}+k N(x) u^{-}=N(x) \varphi^{-} v \quad \text { in } \Omega, \quad u^{-}=0 \quad \text { on } \partial \Omega,
$$

where $\mathcal{L}^{-}, M^{-}$and $\varphi^{-}>0$ are as is Lemma 2.1 and $u^{-}=\varphi^{-} u$. Since $M^{-}<0$ in a neighborhood $\Omega^{1}$ of $\partial \Omega$ and $N>0$ in $\Omega, k_{0}=\sup \left\{M^{-}(x) / N(x): x \in \Omega \backslash \Omega^{1}\right\}$ is well defined, and $k N-M^{-}>0$ in $\Omega$ whenever $k>k_{0}$. Then a standard maximum principle applied to (2.14) ensures uniqueness for that problem, and hence uniqueness for (2.12), if $k>k_{0}$. Still, the strong maximum principle in Appendix B implies that $\partial u^{-} / \partial v<0$ if $v \geqslant 0$ and $v$ is not identically zero. And since $\partial u / \partial v=\partial u^{-} / \partial v$ on $\partial \Omega$ (see (2.3)) the last statement in Proposition 2.5 also follows if $k>k_{0}$. Thus only the existence part remains to be proved. 
After selecting $k_{0}$, we take $\left.k>k_{0}, \delta \in\right] 0, \delta_{0}[$ and rewrite (2.12) as

$$
H(u) \equiv u-G_{1}(u)+k G_{2}(u)=G_{2}(v)
$$

where $G_{1}, G_{2}: C_{0}^{0,1}(\bar{\Omega}) \rightarrow C_{0}^{1, \delta}(\bar{\Omega})$ are then the Green operators $\left(u=G_{1}(v)\right.$ and $u=$ $G_{2}(v)$ ) of the problems (2.7) and $\mathcal{L} u=N(x) v$ in $\Omega, u=0$ on $\partial \Omega$, respectively; note that $G_{1}$ and $G_{2}$ are bounded, according to Proposition 2.3. Since the imbedding $i: C_{0}^{1, \delta}(\bar{\Omega}) \rightarrow C_{0}^{0,1}(\bar{\Omega})$ is compact, $\hat{H}=H \circ i$ is a compact perturbation of the identity in $C_{0}^{1, \delta}(\bar{\Omega})$, and since $k>k_{0}, \hat{H}$ is injective. Thus the standard Riesz theory [21] on compact operators applies and $\hat{H}$ is readily seen to be a linear homeomorphism. Then if $v \in C_{0}^{0,1}(\bar{\Omega}), w=G_{2}(v) \in C_{0}^{1, \delta}(\bar{\Omega})$ and $(2.15)$ has a unique solution $u \in C^{1, \delta}(\bar{\Omega})$ such that $\|u\|_{C^{1, \delta}(\bar{\Omega})} \leqslant K^{\prime}\left\|G_{2}(v)\right\|_{C^{1, \delta}(\bar{\Omega})} \leqslant K\|v\|_{C_{0}^{0,1}(\bar{\Omega})}$ and the estimate (2.13) follows. Also, as in Proposition 2.3, $u \in C^{2}(\Omega)$, and the proof is complete.

Now we are in a position to analyze the linear eigenvalue problem (1.5). This problem was considered by Bertsch and Rostamian [8] for operators in divergence form (see also [28] for an extension to operators in general form) via Hardy-Sobolev inequalities, see Remark 2.4 above. As for the regularity of the eigenfunctions, in [8] it was shown that they are in $C^{2}(\Omega) \cap H_{0}^{1}(\Omega)$ if $M$ and $N$ satisfy assumption (H.3') above with $\alpha>-1$, and in $C^{2}(\Omega) \cap C_{0}^{1}(\bar{\Omega})$ if $\alpha>0$. But the corresponding spectrum coincides with that obtained when the eigenfunctions are required to be in $C^{2}(\Omega) \cap C^{1}(\bar{\Omega})$, as shown in the following theorem, which also provides a fairly complete characterization of the spectrum for operators in general form.

THEOREM 2.6. - Under the assumptions (H.1)-(H.2), (H.3') and (H.4), the spectrum of the linear eigenvalue problem (1.5), with $U \in C^{2}(\Omega) \cap C^{0,1}(\bar{\Omega})$, is such that

(i) It consists (at most) of a countable set of eigenvalues which are isolated, and the eigenfunctions are in $C^{1, \delta}(\bar{\Omega})$ for all $\delta$ such that $0<\delta<\delta_{0}=\min \{\gamma, \alpha+1\}$.

(ii) It contains a unique principal eigenvalue (i.e., a real eigenvalue with an associated eigenfunction in the interior of the positive cone of $C_{0}^{1}(\bar{\Omega})$, namely, such that $U>0$ in $\Omega$ and $\partial U / \partial v<0$ on $\partial \Omega$ ), which is simple.

(iii) The (not necessarily real) eigenvalues of (1.5) are such that

$$
\operatorname{Re} \lambda>\lambda_{1} \quad \text { if } \lambda \neq \lambda_{1}, \text { and } \operatorname{Re} \lambda \geqslant c_{2}+c_{1}|\operatorname{Im} \lambda|,
$$

where $\lambda_{1}$ is the principal eigenvalue of (1.5) and the real constants $c_{1}>0$ and $c_{2}$ are independent of $\lambda$.

(iv) It does not change when the eigenfunctions are only required to be in $C^{2}(\Omega) \cap$ $H_{0}^{1}(\Omega)$, and coincides with the spectrum of the formal adjoint problem (1.7).

Proof. - We subsequently prove the statements (i), (ii) and (iii). Since the operator $\mathcal{L}$ is not necessarily selfadjoint, its spectrum is not necessarily real, and we must work with the complexifications of $\mathcal{L}$ and the various function spaces; this trivial extension will be automatically made below.

(i) If $k_{0}$ is as in Proposition 2.5, $k>k_{0}$ and $0<\delta<\delta_{0}$, then the problem (2.12) defines a Green operator $u=G(v)$, with $G: C_{0}^{0,1}(\bar{\Omega}) \rightarrow C_{0}^{1, \delta}(\bar{\Omega})$ bounded. And if $i$ is the compact imbedding of $C_{0}^{1, \delta}(\bar{\Omega})$ into $C_{0}^{0,1}(\bar{\Omega})$, then $\hat{G}=G \circ i: C_{0}^{1, \delta}(\bar{\Omega}) \rightarrow C_{0}^{1, \delta}(\bar{\Omega})$ is 
compact. This completes the proof of the second statement in part (i). And the first statement follows by the standard spectral theory for compact operators [21], when taking into account that the eigenvalues of (1.5) and $\hat{G}, \mu$, are related by

$$
\mu=1 /(\lambda+k) .
$$

(ii) Let $\hat{G}$ be the compact operator defined above. According to Proposition $2.5, \hat{G}$ maps the positive cone of $C_{0}^{1, \delta}(\bar{\Omega})$ into its interior, and the Krein-Rutman theorem [3, 19] readily implies that $\hat{G}$ has a unique principal eigenvalue $\mu_{1}$, which is simple, strictly positive and such that any other eigenvalue of $\hat{G}$ satisfies $|\mu|<\mu_{1}$. And taking into account the relation (2.17) between the spectra of $\hat{G}$ and (2.4), the statement (ii) readily follows, with $\lambda_{1}=1 / \mu_{1}-k$ (for any $k>k_{0}$ ). Note that, in addition, any other eigenvalue $\lambda$ of (1.5) satisfies $\left[|\operatorname{Re} \lambda+k|^{2}+|\operatorname{Im} \lambda|^{2}\right]^{1 / 2}=|\lambda+k|=1 /|\mu|>1 / \mu_{1}=\left|\lambda_{1}+k\right|$, and since that inequality holds for all $k>k_{0}$, we readily obtain

$$
\operatorname{Re} \lambda \geqslant \lambda_{1} \text {. }
$$

(iii) In order to obtain the first inequality in (2.16) we assume for contradiction that there is an eigenvalue of (1.5), $\lambda \neq \lambda_{1}$, such that $\operatorname{Re} \lambda \leqslant \lambda_{1}$ or, according to (2.18),

$$
\lambda \neq \lambda_{1}, \quad \operatorname{Re} \lambda=\lambda_{1} .
$$

If, in addition, we rewrite the problem (1.5) as in Lemma 2.1, then the problems

$$
\begin{aligned}
& \mathcal{L}^{-} U_{1}^{-}-M^{-}(x) U_{1}^{-}=\lambda_{1} N(x) U_{1}^{-} \quad \text { in } \Omega, \quad U_{1}^{-}=0 \quad \text { on } \partial \Omega, \\
& \mathcal{L}^{-} U^{-}-M^{-}(x) U^{-}=\lambda N(x) U^{-} \quad \text { in } \Omega, \quad U^{-}=0 \quad \text { on } \partial \Omega,
\end{aligned}
$$

possess nontrivial solutions, where $\mathcal{L}^{-}$and $M^{-}$are as in Lemma 2.1. Also, $U_{1}^{-}$can be chosen to be real and such that

$$
U_{1}^{-}>0 \quad \text { in } \Omega, \quad \partial U_{1}^{-} / \partial v<0 \quad \text { on } \partial \Omega,
$$

because $\lambda_{1}$ is a principal eigenvalue. Now, for each $\beta \in \mathbb{R}$, we define

$$
u_{\beta}(x, t)=U^{-} \exp [-(\lambda+k) t]+\text { c.c. }-\beta U_{1}^{-} \exp \left[-\left(\lambda_{1}+k\right) t\right],
$$

where c.c. stands for the complex conjugate. This function is readily seen to satisfy

$$
N(x) \partial u_{\beta} / \partial t+\mathcal{L}^{-} u_{\beta}-M^{-}(x) u_{\beta}+k N(x) u_{\beta}=0 \quad \text { in } \Omega, \quad u=0 \quad \text { on } \partial \Omega
$$

for all $t \in \mathbb{R}$. If we choose the real constant $k$ such that $k>k_{0}$, where $k_{0}$ is as selected in the proof of Proposition 2.5, right after (2.14), then

$$
-M^{-}(x)+k N(x)>0 \text { for all } x \in \Omega .
$$

In addition, the constant $\tilde{\beta}$, given by

$$
\tilde{\beta}=\inf \left\{\beta \in \mathbb{R}: u_{\beta}(x, t)<0 \text { for all } x \in \Omega \text { and all } t \in \mathbb{R}\right\}
$$


is well-defined (see (2.19) and take into account that $U_{1}^{-}, \operatorname{Re} U^{-}$and $\operatorname{Im} U^{-}$are in $C_{0}^{1}(\bar{\Omega})$, and that $u_{\tilde{\beta}} \exp \left[\left(\lambda_{1}+k\right) t\right]$ is periodic in $\left.t\right)$, and such that (a) $u_{\tilde{\beta}}(x, t) \leqslant 0$ for all $x \in \bar{\Omega}$ and all $t \in \mathbb{R}$, and (b) either $u_{\tilde{\beta}}=0$ at some $\left(x_{0}, t_{0}\right) \in \Omega \times \mathbb{R}$, or $\partial u_{\tilde{\beta}} / \partial v=0$ at some $\left(x_{0}, t_{0}\right) \in \partial \Omega \times \mathbb{R}$. Then we only need to take into account that $u_{\tilde{\beta}}$ also satisfies (2.20) for all $t \in \mathbb{R}$, with $M^{-}$and $k N$ satisfying (2.21), and apply the strong maximum principle for parabolic problems with locally bounded coefficients in Appendix B, to get the required contradiction. Thus the first inequality in (2.16) has been obtained.

In order to get the second inequality in (2.16), we multiply (1.5) by $\bar{U}$ (= the complex conjugate of $U$ ), integrate in $\Omega$, integrate by parts and take the real and imaginary parts of the resulting equation, to obtain

$$
\begin{aligned}
& 2(\operatorname{Re} \lambda) \int_{\Omega} N|U|^{2} d x+2 \int_{\Omega} M|U|^{2} d x \\
& =2 \sum \int_{\Omega} a_{i j} \frac{\partial U}{\partial x_{i}} \frac{\partial \bar{U}}{\partial x_{j}} d x+\sum \int_{\Omega}\left(b_{i}+\sum \frac{\partial a_{i j}}{\partial x_{j}}\right)\left(\bar{U} \frac{\partial U}{\partial x_{i}}+U \frac{\partial \bar{U}}{\partial x_{i}}\right) d x, \\
& 2(\operatorname{Im} \lambda) \int_{\Omega} N|U|^{2} d x=\sum \int_{\Omega}\left(b_{i}+\sum \frac{\partial a_{i j}}{\partial x_{j}}\right)\left(\bar{U} \frac{\partial U}{\partial x_{i}}-U \frac{\partial \bar{U}}{\partial x_{j}}\right) d x .
\end{aligned}
$$

Now, since the operator $-\mathcal{L}$ is uniformly, strongly elliptic in $\Omega$, there is a constant $k_{0}>0$ (independent of $U$ ) such that

$$
\sum \int_{\Omega} a_{i j} \frac{\partial U}{\partial x_{i}} \frac{\partial \bar{U}}{\partial x_{j}} d x \geqslant k_{0} \int_{\Omega}|\nabla U|^{2} d x .
$$

Also, since $U=0$ on $\partial \Omega$, we can use the standard Hardy-Sobolev inequality [13] to obtain

$$
\int_{\Omega}|U(x)|^{2} d(x)^{-2} d x \leqslant c_{0} \int_{\Omega}|\nabla U|^{2} d x,
$$

where the constant $c_{0}$ is independent of $U$ and $d(x)$ is the distance from $x$ to $\partial \Omega$, as above. But, according to assumptions (H.1)-(H.2), (H.3') and (H.4), there is a subdomain $\Omega_{2} \subset \Omega\left(\partial \Omega_{2} \subset \Omega\right)$ and two constants, $k_{1}$ and $k_{2}$, such that

$$
\begin{aligned}
& |M(x)| d(x)^{2}<k_{0} /\left(4 c_{0}\right), \quad\left|\sum\left(b_{i}+\sum \partial a_{i j} / \partial x_{j}\right)\right|^{2} d(x)^{2}<k_{0}^{2} /\left(16 c_{0}\right), \\
& \quad \text { if } x \in \Omega \backslash \Omega_{2}, \\
& |M(x)| / N(x)<k_{1}, \quad\left|\sum\left(b_{i}+\sum \partial a_{i j} / \partial x_{j}\right)\right|^{2} / N(x)<k_{2}, \quad \text { if } x \in \Omega_{2} .
\end{aligned}
$$

From these inequalities and (2.25) we subsequently obtain

$$
\begin{aligned}
\left.\left|\int_{\Omega} M\right| U\right|^{2} d x \mid & <\left[k_{0} /\left(4 c_{0}\right)\right] \int_{\Omega}|U|^{2} d(x)^{-2} d x+k_{1} \int_{\Omega} N|U|^{2} d x \\
& \leqslant\left(k_{0} / 4\right) \int_{\Omega}|\nabla U|^{2} d x+k_{1} \int_{\Omega} N|U|^{2} d x
\end{aligned}
$$


and, by a similar argument and Hölder inequality,

$$
\begin{aligned}
& \left|\sum \int_{\Omega}\left(b_{i}+\sum \frac{\partial a_{i j}}{\partial x_{j}}\right)\left(\bar{U} \frac{\partial U}{\partial x_{i}} \pm U \frac{\partial \bar{U}}{\partial x_{i}}\right) d x\right| \\
& \quad \leqslant\left(k_{0} / 2\right) \int_{\Omega}|\nabla U|^{2} d x+\left(8 / k_{0}\right)\left[\left(k_{0}^{2} / 16\right) \int_{\Omega}|\nabla U|^{2} d x+k_{2} \int_{\Omega} N|U|^{2} d x\right] \\
& \quad=k_{0} \int_{\Omega}|\nabla U|^{2} d x+\left(8 k_{2} / k_{0}\right) \int_{\Omega} N|U|^{2} d x .
\end{aligned}
$$

When using (2.24) and (2.26)-(2.27) in (2.22)-(2.23) we obtain

$$
\begin{aligned}
& (\operatorname{Re} \lambda) \int_{\Omega} N|U|^{2} d x>\left(3 k_{0} / 4\right) \int_{\Omega}|\nabla U|^{2} d x-\left(k_{1}+4 k_{2} / k_{0}\right) \int_{\Omega} N|U|^{2} d x, \\
& (\operatorname{Im} \lambda) \int_{\Omega} N|U|^{2} d x<\left(k_{0} / 2\right) \int_{\Omega}|\nabla U|^{2} d x+\left(4 k_{2} / k_{0}\right) \int_{\Omega} N|U|^{2} d x,
\end{aligned}
$$

and the second estimate in (2.16) readily follows.

(iv) Since Proposition 2.5 applies also to the adjoint eigenvalue problem (1.7), we can choose a constant $k>0$ sufficiently large such that, for each $V \in C_{0}^{0,1}(\bar{\Omega})$, the problems

$$
\begin{aligned}
& \mathcal{L} U-M(x) U+k N(x) U=N(x) V \quad \text { in } \Omega, \quad U=0 \quad \text { on } \partial \Omega, \\
& \mathcal{L}^{*} U-M(x) U+k N(x) U=N(x) V \quad \text { in } \Omega, \quad U=0 \quad \text { on } \partial \Omega,
\end{aligned}
$$

possess a unique solution. Since these two problems are formally adjoint of each other, their Green operators, are readily seen to be adjoint of each other in the pre-Hilbert space, $X=\left(C^{1}(\bar{\Omega}),\langle\cdot, \cdot\rangle\right)$, where the inner product $\langle\cdot, \cdot\rangle$ is defined as

$$
\left\langle U_{1}, U_{2}\right\rangle \equiv \int_{\Omega} N(x) U_{1}(x) U_{2}(x) d x .
$$

Now, let $G$ and $G^{*}$ be the Green operators of (2.29) and (2.30). If we multiply (2.29) by $\mathrm{U}$, integrate in $\Omega$, apply integration by parts and proceed as in the proof of part (iii) above, we obtain

$$
\left(3 k_{0} / 2\right) \int_{\Omega}|\nabla U|^{2} d x+\left(2 k-1-2 k_{1}-8 k_{2} / k_{0}\right) \int_{\Omega} N|U|^{2} d x<\int_{\Omega} N|V|^{2} d x,
$$

where the constants $k_{0}, k_{1}$ and $k_{2}$ are as in (2.28). If we choose $k$ such that $k \geqslant$ $1 / 2+k_{1}+4 k_{2} / k_{0}$, then this inequality shows that

$$
\|G(V)\|_{H^{1}(\Omega)}^{2} \leqslant K\|V\|_{X}^{2},
$$

with the constant $K$ independent of $V$; and since, according to Hardy-Sobolev inequalities [13] and (compact) imbedding theorems [1], the imbedding of $H_{0}^{1}(\Omega)$ into the completion of $X$ is compact, the Green operator $G$ is compact in $H_{0}^{1}(\Omega)$, and its 
continuous extension is compact in the completion of $X$. Similarly, the adjoint operator $G^{*}$ is compact in $X$. Then we only need to apply [21, $\left.\$ 11.4 .2, \S 11.5 .5\right]$ and take into account that if $\mu$ is in the spectrum of $G$ then $\bar{\mu}$ is also in the spectrum (because $G$ is real) to obtain that the spectra of $G$ in $H_{0}^{1}(\Omega)$ and in $C_{0}^{1}(\bar{\Omega})$ coincide with the spectrum of the continuous extension of $G$ to the completion of $X$, and with that of $G^{*}$. And we only need to take into account that the eigenvalues of (1.5) and (1.7) are obtained from the eigenvalues $\mu$ of $G$ and $G^{*}$, respectively, by means of (2.17) to obtain the two statements in property (iv). Thus the proof of the theorem is complete.

Note that, according to Theorem 2.6, the spectrum of the linear eigenvalue problem (1.5) exhibits similar properties as the spectra of related regular (i.e., with smooth coefficients in $\bar{\Omega}$ ) second-order elliptic problems, except for the last estimate in statement (iii), which is asymptotically (as $|\lambda| \rightarrow \infty$ ) sharper in the regular case (when $\operatorname{Re} \lambda \geqslant$ $c_{1}+c_{2}|\operatorname{Im} \lambda|^{2}$, with $c_{2}>0$ ).

Now we prove that the min-max characterization of the principal eigenvalue introduced in [22] also applies when the coefficients of $\mathcal{L}$ and the function $M$ satisfy (H.2), (H.3') and (H.4) (Proposition 2.8 below). The idea of the proof follows that of [7]. The following previous characterization is needed.

LEMMA 2.7. - Under the assumptions (H.1), (H.2), (H.3') and (H.4), the problem (1.5) possesses a strictly positive principal eigenvalue if and only if the operator $\mathcal{L}-M(x)$ satisfies the strong maximum principle, i.e. if $v \in C^{2}(\Omega) \cap C^{1}(\bar{\Omega})$ is such that

$$
v \neq 0, \quad \mathcal{L} v-M(x) v \geqslant 0 \quad \text { in } \Omega, \quad v \geqslant 0 \quad \text { on } \partial \Omega,
$$

then $v>0$ for all $x \in \Omega$ and $\partial v(x) / \partial v<0$ (where $v$ is the outward unit normal, as above) for all $x \in \partial \Omega$ such that $v(x)=0$.

Proof. - If $\mathcal{L}-M(x)$ satisfies a strong maximum principle then the principal eigenvalue of (1.5), $\lambda_{1}$, exists (Theorem 2.6) and is readily seen to be strictly positive. In order to prove the converse we assume without loss of generality (Lemma 2.1) that $M(x) \leqslant 0$ in a neighborhood $\Omega^{1}$ of $\partial \Omega$. For each function $v$ satisfying (2.33), we consider the function

$$
w=v+\varepsilon+\varepsilon k U_{1}
$$

where $\varepsilon>0, U_{1}>0$ is an eigenfunction of (1.5) associated with the principal eigenvalue $\lambda_{1}$ and $k=\sup \left\{2|M(x)| /\left[\lambda_{1} N(x) U_{1}(x)\right]: x \in \Omega \backslash \Omega^{1}\right\}$. Then

$$
\mathcal{L} w-M(x) w \geqslant \varepsilon\left[k \lambda_{1} N(x) U_{1}(x)-M(x)\right]>0 \text { in } \Omega .
$$

Moreover, since $v$ is continuous, for each $\varepsilon>0$ there is a constant $\gamma(\varepsilon)>0$ such that $w>0$ in $\Omega_{\varepsilon}=\{x \in \Omega: d(x)<\gamma(\varepsilon)\}$; and $w>0$ in $\Omega \backslash \Omega_{\varepsilon}$, as readily seen upon application of the generalized maximum principle to (2.35) in $\Omega \backslash \Omega_{\varepsilon}$ (note that $U_{1}$ satisfies (2.33), with strict inequalities in $\Omega \backslash \Omega_{\varepsilon}$ ). Thus $w>0$ in $\Omega$ for all $\varepsilon>0$, and by letting $\varepsilon \rightarrow 0$, we obtain $v \geqslant 0$ in $\Omega$. Thus, the generalized maximum principle and the strong maximum principle in Appendix B yield $v>0$ in $\Omega$ and $\partial v(x) / \partial v<0$ if $x \in \partial \Omega$ and $v(x)=0$; and the proof is complete. 
Proposition 2.8. - Under the assumptions (H.1), (H.2), (H.3') and (H.4), the principal eigenvalue of (1.5) is given by

$$
\lambda_{1}=\sup \{\inf \{[\mathcal{L} v-M(x) v] /[N(x) v]: x \in \Omega\}: v \in \mathcal{P}\},
$$

where $\mathcal{P}$ is the set of those functions of $C^{2}(\Omega) \cap C_{0}^{1}(\bar{\Omega})$ such that $v>0$ in $\Omega$.

Proof. - Let $\tilde{\lambda}_{1}$ be the principal eigenvalue of (1.5) and let $U_{1}>0$ be an associated eigenfunction. Then $v=U_{1} \in \mathcal{P}$ and satisfies $\mathcal{L} v-M(x) v=\tilde{\lambda}_{1} N(x) v \geqslant \lambda N(x) v$ in $\Omega$ whenever $\lambda \leqslant \tilde{\lambda}_{1}$. This shows that the set $\{\inf \{[\mathcal{L} v-M(x) v] /[N(x) v]: x \in \Omega\}: v \in \mathcal{P}\}$ contains the interval $\left.]-\infty, \tilde{\lambda}_{1}\right]$. Thus $((2.36)$ is well defined and $) \tilde{\lambda}_{1} \leqslant \lambda_{1}(\leqslant \infty)$.

Now we prove that the inequality $\tilde{\lambda}_{1}<\lambda_{1}$ cannot be satisfied. Assume for contradiction that there is a real number $\varepsilon>0$ and a function $v \in \mathcal{P}$ such that $\tilde{\lambda}_{1}+\varepsilon<$ $\inf \{[\mathcal{L} v-M(x) v] /[N(x) v]: x \in \Omega\}$, and let $U_{1}>0$ be an eigenfunction associated with $\tilde{\lambda}_{1}$. Let the constant $\beta_{3} \geqslant 0$ be defined as $\beta_{3}=\min \left\{\beta_{1}, \beta_{2}\right\}$, where $\beta_{1}=\sup \{\beta \in$ $\mathbb{R}^{+}: v-\beta U_{1} \geqslant 0$ in $\left.\Omega\right\}, \beta_{2}=\sup \left\{\beta \in \mathbb{R}^{+}: \partial\left(v-\beta U_{1}\right) / \partial v \leqslant 0\right.$ on $\left.\partial \Omega\right\}$ and let $w$ be the real function $w=v-\beta_{3} U_{1}$. Then $w \geqslant 0$ in $\Omega$ and either $w=0$ at some point of $\Omega$ (if $\beta_{3}=\beta_{1}$ ) or $w=\partial w / \partial v=0$ at some point of $\partial \Omega$ (if $\beta_{3}=\beta_{2}$ ). On the other hand,

$$
\mathcal{L} w-M(x) w-\left(\tilde{\lambda}_{1}-\varepsilon\right) N(x) w>\varepsilon N(x)(v+w)>0 \quad \text { in } \Omega,
$$

and this is in contradiction with the result in Lemma 2.7. Thus the proof is complete.

The following result deals with the dependence of the principal eigenvalue of (1.5) on the coefficient of $U$ in the left hand side of (1.5).

Proposition 2.9. - Let $\Omega, \mathcal{L}, M$, and $N$ satisfy assumptions (H.1)-(H.2), (H.3') and (H-4), and let $M_{1}$ be a nonzero function that is non-negative in $\Omega$ and satisfies (H.3'). For each $\mu \in \mathbb{R}$, let $\lambda=\Lambda(\mu)$ be the principal eigenvalue of

$$
\left[\mathcal{L}-M(x)+\mu M_{1}(x)\right] u=\lambda N(x) u \quad \text { in } \Omega, \quad u=0 \quad \text { on } \partial \Omega,
$$

with $u \in C^{2}(\Omega) \cap C^{1}(\bar{\Omega})$. Then the function $\Lambda: \mathbb{R} \rightarrow \mathbb{R}$ is analytic, strictly increasing and concave.

Proof. - In order to prove that the function $\Lambda$ is analytic in a neighborhood of each $\mu_{0} \in \mathbb{R}$, we only need (as in [17]) to apply the implicit function theorem to the system

$$
G(u, \lambda, \mu) \equiv u-G_{0}(u)+\mu G_{1}(u)-\lambda G_{2}(u)=0, \quad \int_{\Omega} u_{0} u d x=1,
$$

where $u_{0}>0$ is an eigenfunction of (2.37) at $\mu=\mu_{0}, \lambda=\lambda_{0} \equiv \Lambda\left(\mu_{0}\right)$, such that $\int_{\Omega} u_{0}^{2} d x=1, G_{0}$ is the Green operator of (2.7) and $G_{1}$ and $G_{2}$ are the Green operators of the problems obtained from (2.7) when replacing $M$ by $M_{1}$ and $N$ respectively. Note that the first equation in (2.38) is equivalent to (2.37), and that $G_{0}, G_{1}, G_{2}: C_{0}^{1}(\bar{\Omega}) \rightarrow C_{0}^{1}(\bar{\Omega})$ are linear and continuous (Proposition 2.3); thus $G: C_{0}^{1}(\bar{\Omega}) \times \mathbb{R}^{2} \rightarrow C_{0}^{1}(\bar{\Omega})$ is analytic. Still, $\lambda_{0}^{\prime} \equiv \Lambda^{\prime}\left(\mu_{0}\right)$ and $u_{0}^{\prime} \equiv d u\left(\mu_{0}\right) / d \mu$ satisfy $u_{0}^{\prime}-G_{0}\left(u_{0}^{\prime}\right)+\mu_{0} G_{1}\left(u_{0}^{\prime}\right)-\lambda_{0} G_{2}\left(u_{0}^{\prime}\right)=$ 
$\lambda_{0}^{\prime} G_{2}\left(u_{0}\right)-G_{1}\left(u_{0}\right)$ (as seen upon differentiation in (2.38)), and this equation is equivalent to

$$
\begin{aligned}
& {\left[\mathcal{L}-M(x)+\mu_{0} M_{1}(x)-\lambda_{0} N(x)\right] u_{0}^{\prime}=\left[\lambda_{0}^{\prime} N(x)-M_{1}(x)\right] u_{0} \quad \text { in } \Omega,} \\
& u_{0}^{\prime}=0 \text { on } \partial \Omega .
\end{aligned}
$$

The solvability of this singular, linear problem requires that

$$
\lambda_{0}^{\prime} \int_{\Omega} N(x) u_{0} u_{0}^{*} d x=\int_{\Omega} M_{1}(x) u_{0} u_{0}^{*} d x,
$$

where $u_{0}^{*}>0$ is an eigenfunction of the adjoint, linearized problem

$$
\left[\mathcal{L}^{*}-M(x)+\mu_{0} M_{1}(x)\right] u_{0}^{*}=\lambda_{0} N(x) u_{0}^{*} \quad \text { in } \Omega, \quad u_{0}^{*}=0 \quad \text { on } \partial \Omega,
$$

with the operator $\mathcal{L}^{*}$ as defined in (1.3). Note that $\lambda_{0}$ is also the principal eigenvalue of (2.41) (Theorem 2.6 (iii)-(iv)). Since, in addition, $N>0$ in $\Omega, M_{1} \geqslant 0$ in $\Omega$ and $M_{1}$ is not identically zero, (2.40) implies that $\lambda_{0}^{\prime} \equiv d \Lambda\left(\mu_{0}\right) / d \mu>0$. And since $\mu_{0}$ was arbitrary, the function $\Lambda$ is strictly increasing, as stated. Finally, the proof that $\Lambda$ is concave is identical to that in [7, Proposition 2.1], and the proof is complete.

Now we consider the existence of a principal eigenvalue of (1.5) when $N$ vanishes in a subset of $\Omega$.

THEOREM 2.10. - Let $\Omega, \mathcal{L}$ and $M$ be as in assumptions (H.1)-(H.2), (H.3'), and let $N$ satisfy (H.3') and be such that $N=0$ in $C$ and $N>0$ in $\Omega \backslash C$, where the closed set $C$ is such that $\emptyset \neq C \subset \Omega$. Then (1.5) possesses a principal eigenvalue if and only if the quantity

$$
\mu_{0}=\sup \left\{\inf \left\{[\mathcal{L} v-M(x) v] / v: x \in \Omega^{\prime}\right\}: \Omega^{\prime} \in S, v \in \mathcal{P}\right\} \leqslant \infty
$$

is strictly positive, where $S$ is the set of those open subsets of $\Omega$ such that $C \subset \Omega^{\prime}$ and $\partial \Omega \subset \partial \Omega^{\prime}$ (that is, $\Omega^{\prime}$ is an open neighborhood of both $C$ and $\partial \Omega$ ), and $\mathcal{P}$ is the set of those functions $v \in C^{2}(\Omega) \cap C^{1}(\bar{\Omega})$ such that $v>0$ in $\Omega$. Also, if $\mu_{0}>0$ then the principal eigenvalue of $(1.5)$ is unique and simple.

Proof. - Let $N_{1}$ be a function satisfying (H.3') and such that

$$
N_{1}(x) \geqslant 1+[1+N(x)] / d(x)^{\varepsilon} \quad \text { in } \Omega \text {, for some } \varepsilon>0,
$$

where $d(x)$ is the distance from $x$ to $\partial \Omega$, as above, and for each $\lambda \in \mathbb{R}$ let $\mu=\mathcal{M}(\lambda)$ be the (unique) principal eigenvalue of

$$
\mathcal{L} U-M(x) U-\lambda N(x) U=\mu N_{1}(x) U \quad \text { in } \Omega, \quad U=0 \quad \text { on } \partial \Omega,
$$

which is simple (Theorem 2.6). Note that (1.5) has a principal eigenvalue $\lambda$ if and only if $\mathcal{M}(\lambda)=0$. Since, in addition, the function $\mu=\mathcal{M}(\lambda)$ is analytic, strictly decreasing and concave (Proposition 2.9), $\mathcal{M}(\lambda) \rightarrow-\infty$ as $\lambda \rightarrow \infty$, and the stated result readily 
follows from the following property, which is proved below: the quantity $\mu_{0} \leqslant \infty$ defined in (2.42) is strictly positive if and only $\mathcal{M}(\lambda)>0$ for some $\lambda \in \mathbb{R}$.

If $\mu=\mathcal{M}(\lambda)>0$ for some $\lambda \in \mathbb{R}$ and $U>0$ is an associated eigenfunction of (2.44), then $U \in \mathcal{P}$ and

$$
(\mathcal{L} U-M(x) U) / U \geqslant \mu N_{1}(x)+\lambda N(x) \text { in } \Omega .
$$

But, according to (2.43) the right hand side of (2.45) is larger than $\mu / 2>0$ in $\Omega^{\prime}=$ $\Omega \cap\left(\Omega_{1}^{\prime} \cup \Omega_{2}^{\prime}\right)$, where $\Omega_{1}^{\prime}$ and $\Omega_{2}^{\prime}$ are appropriate open neighborhoods of $C$ and $\partial \Omega$ respectively. Then $\mu_{0} \geqslant \mu / 2>0$.

And conversely, if $\mu_{0}>0$ then there is a function $v \in C^{2}(\Omega) \cap C^{1}(\bar{\Omega})$ such that $v>0$ in $\Omega$ and $[\mathcal{L} v-M(x) v] / v>k>0$ in $\Omega^{\prime}$, for some $\Omega^{\prime} \in S$. And if, without loss of generality we assume that $M<0$ in a neighborhood of $\partial \Omega$ (Lemma 2.1) and $\varepsilon_{0}>0$ is sufficiently small, then $v_{0}=v+\varepsilon_{0}$ still satisfies

$$
\mathcal{L} v_{0}-M(x) v_{0}>0 \quad \text { in } \Omega^{\prime} \quad \text { and } v_{0}>0 \quad \text { in } \bar{\Omega} .
$$

Let us rewrite (2.44) in terms of $V=U / v_{0}$ as

$$
\begin{aligned}
& \mathcal{L} V+\sum \tilde{b}_{i}(x) \partial V / \partial x_{i}+[\tilde{M}(x)-\lambda N(x)] V=\mu N_{1}(x) V \quad \text { in } \Omega, \\
& V=0 \quad \text { on } \partial \Omega,
\end{aligned}
$$

where

$$
\tilde{b}_{i}=\left(2 / v_{0}\right) \sum a_{i j} \partial v_{0} / \partial x_{j}, \quad \tilde{M}(x)=\left[\mathcal{L} v_{0}-M(x) v_{0}\right] / v_{0} .
$$

Now $\tilde{M}>0$ in $\Omega^{\prime}\left(\right.$ see (2.46)) and $\tilde{M}$ and $1 / N$ are bounded in $\Omega \backslash \Omega^{\prime}$; then $\tilde{M}-\lambda N>0$ in $\Omega$ for some $\lambda \in \mathbb{R}$. Since, in addition, $V=U / v_{0}>0$ in $\Omega$, standard maximum principles applied to (2.47) readily imply that $\mu=\mathcal{M}(\lambda)>0$. Thus the proof is complete.

Remark 2.11. - Under the assumptions of Theorem 2.10, the existence of a principal eigenvalue is determined by the sign of the quantity $\mu_{0}$, defined in (2.42). That quantity is now calculated in several cases that have been already considered in the literature [34] for equations with bounded coefficients. Thus we generalize these results to our singular case.

(A) [34, Theorem 6.2]. If $C=\bar{\Omega}_{0} \subset \Omega$, where $\Omega_{0}=\Omega \backslash\left(\bigcup_{j=1}^{h} \bar{\Omega}_{j}\right)$, with $\bar{\Omega}_{i} \cap \bar{\Omega}_{j}=\emptyset$ for $i \neq j$ and $\Omega_{1}, \ldots, \Omega_{h}$ are subdomains of $\Omega$ with $C^{2, \gamma}$-boundaries, then the quantity $\mu_{0}$ defined in (2.42) is the principal eigenvalue of

$$
\mathcal{L} U-M(x) U=\mu U \quad \text { in } \Omega_{0}, \quad U=0 \quad \text { on } \partial \Omega_{0} .
$$

Since $\Omega_{0} \subset \Omega^{\prime}$ for all $\Omega^{\prime} \in S$, the result in Proposition 2.8 readily implies that the principal eigenvalue of $(2.48), \tilde{\mu}$, is such that $\tilde{\mu} \geqslant \mu_{0}$. In order to see that, conversely, $\tilde{\mu} \leqslant \mu_{0}$, we assume (without loss of generality, Lemma 2.1 ) that $M<0$ in a neighborhood of $\partial \Omega$. Let $U>0$ (in $\Omega_{0}$ ) be an eigenfunction of (2.48) associated with $\tilde{\mu}$, let $k_{1}=1+|\tilde{\mu}|+|\max \{M(x): x \in \Omega\}|$, let $V \in C^{2}\left(\Omega_{0}\right) \cap C_{0}^{1}\left(\bar{\Omega}_{0}\right)$ be the unique 
solution of $\mathcal{L} V=k_{1}$ in $\Omega_{0}, V=0$ on $\partial \Omega_{0}$, and let $k_{2}=\sup \left\{\left[k_{1} V(x)-1\right] / U(x): x \in \Omega\right\}$. Then the function $U_{\varepsilon}$, defined as $U_{\varepsilon}=U+\varepsilon(1+V)$ satisfies

$$
\left(\mathcal{L} U_{\varepsilon}-M(x) U_{\varepsilon}\right) / U_{\varepsilon}>\tilde{\mu}-k_{2} \varepsilon \quad \text { in } \Omega_{0}, \quad U_{\varepsilon}>0 \quad \text { in } \bar{\Omega}_{0},
$$

for all sufficiently small $\varepsilon>0$. Also, since $M$ and the coefficients of $\mathcal{L}$ are smooth in $\partial \Omega_{0} \backslash \partial \Omega, U$ (and thus $U_{\varepsilon}$ ) is of class $C^{2}$ up to $\partial \Omega_{0} \backslash \partial \Omega$, and $U_{\varepsilon}$ can be extended to $\Omega$ as a $C^{2}$-function, still called $U_{\varepsilon}$, which is strictly positive in $\bar{\Omega}$. Since, in addition, $\left(\mathcal{L} U_{\varepsilon}-M(x) U_{\varepsilon}\right) / U_{\varepsilon}$ is continuous in $\partial \Omega_{0} \backslash \partial \Omega$ and satisfies (2.49), we have $\left(\mathcal{L} U_{\varepsilon}-M(x) U_{\varepsilon}\right) / U_{\varepsilon}>\tilde{\mu}-2 k_{2} \varepsilon$ in $\Omega^{\prime}=\Omega \cap \Omega_{0}^{\prime}$, where $\Omega_{0}^{\prime}$ is an open neighborhood of $\Omega_{0}$. Thus $\mu_{0}>\tilde{\mu}-2 k_{2} \varepsilon$ (see (2.42)) and since that inequality holds for all sufficiently small $\varepsilon>0$, we have $\mu_{0} \geqslant \tilde{\mu}$ as stated.

(B) [34, Theorem 6.7]. As a dual case of that in A, let us assume that $C=\bigcup_{j=1}^{h} \bar{\Omega}_{j} \subset$ $\Omega$, where $\Omega_{1}, \ldots, \Omega_{h}$ are domains with a smooth boundary, and $\bar{\Omega}_{i} \cap \bar{\Omega}_{j}=\emptyset$ for $i \neq j$. Then the quantity $\mu_{0}$ defined in (2.42)is given by $\mu_{0}=\tilde{\mu} \equiv \min \left\{\tilde{\mu}_{j}: 1 \leqslant j \leqslant h\right\}$ where $\tilde{\mu}_{j}$ is the principal eigenvalue of

$$
\mathcal{L} U_{j}-M(x) U_{j}=\mu_{j} U_{j} \quad \text { in } \Omega_{j}, \quad U_{j}=0 \quad \text { on } \partial \Omega_{j} .
$$

As in the proof of property A above, the definition (2.42) and Proposition 2.8 readily imply that $\mu_{0} \leqslant \tilde{\mu}_{j}$ for $j=1, \ldots, h$ and thus $\mu_{0} \leqslant \tilde{\mu}$. And, in order to prove that, conversely, $\mu_{0} \geqslant \tilde{\mu}$, we again assume, without loss of generality, that

$$
-M(x) \geqslant k / d(x)^{1-\alpha} \quad \text { in a neighborhood of } \partial \Omega,
$$

for some $k>0$ (Lemma 2.1). Also, if $U \in C^{2}(C)$ is defined as $U(x)=U_{j}(x)$ in $\Omega_{j}$, where $U_{j}>0$ is an eigenfunction associated with $\tilde{\mu}_{j}$, then as above a function $U_{\varepsilon}$ can be defined that satisfies (2.49) with $\Omega_{0}=C$ for all sufficiently small $\varepsilon$. Also, as above, there is a strictly positive, $C^{2}$-extension of $U_{\varepsilon}$ to $\bar{\Omega}$, which is such that $\left(\mathcal{L} U_{\varepsilon}-M(x) U_{\varepsilon}\right) / U_{\varepsilon}>\tilde{\mu}-2 k_{2} \varepsilon$ in an open neighborhood $\Omega_{1}^{\prime} \subset \Omega$ of $C$, for some constant $k_{2}$; and, according to (2.51), that inequality also holds in $\Omega_{2}^{\prime}=\Omega \cap \Omega_{2}^{\prime \prime}$, where $\Omega_{2}^{\prime \prime}$ is an open neighborhood of $\partial \Omega$. Since $\Omega^{\prime}=\Omega_{1}^{\prime} \cup \Omega_{2}^{\prime} \in S$, we have $\mu_{0}>\tilde{\mu}-2 k_{2} \varepsilon$ for all sufficiently small $\varepsilon>0$. Thus $\mu_{0} \geqslant \tilde{\mu}$ and the stated property follows.

(C) [34, Theorem 6.4]. If $C$ has measure zero, and $\mu_{0}$ is as defined in (2.42), then $\mu_{0}=\infty$. Thus, in this case (1.5) always has a principal eigenvalue.

In order to prove that $\mu_{0}=\infty$ we may assume (as above, without loss of generality) that the function $M$ satisfies (2.51). Also, since $C$ and $\partial \Omega$ are of measure zero, for each $\varepsilon>0$ there are two subdomains $\Omega_{\varepsilon}^{\prime}, \Omega_{\varepsilon} \subset \Omega$ that satisfy assumption (H.1) and

$$
C \subset \Omega_{\varepsilon}^{\prime} \subset \bar{\Omega}_{\varepsilon}^{\prime} \subset \Omega_{\varepsilon}, \quad \partial \Omega \subset \partial \Omega_{\varepsilon}^{\prime} \quad \text { and } \quad\left|\Omega_{\varepsilon}\right|<\varepsilon
$$

where $\left|\Omega_{\varepsilon}\right|$ is the measure of $\Omega_{\varepsilon}$. Let $\mu_{\varepsilon}$ be the principal eigenvalue of

$$
\mathcal{L} U-M(x) U=\mu_{\varepsilon} U \quad \text { in } \Omega_{\varepsilon}, \quad U=0 \quad \text { on } \partial \Omega_{\varepsilon},
$$


and let $U>0$ be an associated eigenfunction, which is in $C^{2}\left(\Omega_{\varepsilon}\right) \cap C_{0}^{1}\left(\bar{\Omega}_{\varepsilon}\right)$. Then $U>0$ and $[\mathcal{L} U-M(x) U] / U=\mu_{\varepsilon}$ in $\bar{\Omega}_{\varepsilon}^{\prime}$, and $U$ is of class $C^{2}$ in a neighborhood of $\partial \Omega_{\varepsilon}^{\prime} \backslash \partial \Omega$; then $U$ can extended from $\bar{\Omega}_{\varepsilon}^{\prime}$ to $\Omega$ as a strictly positive, $C^{2}(\Omega) \cap C_{0}^{1}(\bar{\Omega})$-function, and from the definition (2.42) we have

$$
\mu_{0} \geqslant \mu_{\varepsilon} .
$$

If we multiply the equation in (2.53) by $U$, integrate in $\Omega_{\varepsilon}$ and integrate by parts then we obtain

$$
\begin{aligned}
\mu_{\varepsilon} \int_{\Omega_{\varepsilon}} U^{2} d x \geqslant & \sum \int_{\Omega_{\varepsilon}}\left[k_{0}\left(\partial U / \partial x_{i}\right)^{2}+\left(b_{i}+\sum \partial a_{i j} / \partial x_{j}\right) U \partial U / \partial x_{i}\right] d x \\
& -\int_{\Omega_{\varepsilon}} M(x) U^{2} d x \\
\geqslant & \left(k_{0} / 2\right) \sum \int_{\Omega_{\varepsilon}}\left(\partial U / \partial x_{i}\right)^{2} d x-\int_{\Omega_{\varepsilon}} \tilde{M}(x) U^{2} d x,
\end{aligned}
$$

where $k_{0}>0$ is the ellipticity constant of $\mathcal{L}$ (i.e., such that $\sum a_{i j}(x) \xi^{i} \xi^{j} \geqslant k_{0}|\xi|^{2}$ for all $x \in \Omega$ and all $\xi \in \mathbb{R}^{n}$ ) and

$$
\tilde{M} \equiv M(x)+\left(2 k_{0}\right)^{-1} \sum\left(b_{i}+\sum \partial a_{i j} / \partial x_{j}\right)^{2} .
$$

Now, according to assumptions (H.2), (H.3') and (2.51), $\tilde{M}$ is bounded above by a constant $k_{1}$ and thus

$$
\mu_{\varepsilon} \geqslant k_{0}\left[\sum \int_{\Omega_{\varepsilon}}\left(\partial U / \partial x^{i}\right)^{2} d x\right] /\left[2 \int_{\Omega_{\varepsilon}} U^{2} d x\right]-k_{1} \geqslant k_{0} \lambda_{\varepsilon} / 2-k_{1},
$$

where $-\lambda_{\varepsilon}$ is the principal eigenvalue of the Laplacian operator $\Delta$ in $\Omega_{\varepsilon}$ (with Dirichlet boundary conditions). But, according to a well-known result by Faber [23] and Krahn [31] $\lambda_{\varepsilon} \geqslant \lambda_{\varepsilon}^{\prime}$, where $\lambda_{\varepsilon}^{\prime}$ is the first eigenvalue of $-\Delta$ in the ball (of $\mathbb{R}^{n}$ ) of measure $\left|\Omega_{\varepsilon}\right|$. A straightforward calculation readily shows that $\lambda_{\varepsilon}^{\prime} \rightarrow \infty$ as $\left|\Omega_{\varepsilon}\right| \rightarrow 0$, i.e., as $\varepsilon \rightarrow 0$ (see (2.52)). Thus $\mu_{\varepsilon} \rightarrow \infty$ as $\varepsilon \rightarrow 0$ and (2.54) implies that $\mu_{0}=\infty$, as stated.

Now we consider the principal eigenvalues of (1.5) when the function $N$ changes sign in $\Omega$. For convenience, we consider the principal eigenvalue $\mu$ of the problem

$$
\mathcal{L} U-M(x) U-\lambda N(x) U=\mu U \quad \text { in } \Omega, \quad U=0 \quad \text { on } \partial \Omega,
$$

for varying values of $\lambda \in \mathbb{R}$. Note that $\mu$ is also the principal eigenvalue of the adjoint problem

$$
\mathcal{L}^{*} U-M(x) U-\lambda N(x) U=\mu U \quad \text { in } \Omega, \quad U=0 \quad \text { on } \partial \Omega,
$$

where $\mathcal{L}^{*}$ is given in (1.3) (Theorem 2.6). 
THEOREM 2.12. - Let $\Omega, \mathcal{L}$ and $M$ be as in assumptions (H.1),(H.2) and (H.3'), and let $N$ satisfy assumption (H.3') and be such that $N>0$ and $N<0$ in two non void, open subsets of $\Omega$. Then:

(i) If, for some $\lambda=\lambda_{0}$, the principal eigenvalue of (2.55) is strictly positive, then (1.5) has exactly two principal eigenvalues, $\lambda_{1}$ and $\lambda_{2}$, which are such that $\lambda_{1}<\lambda_{0}<\lambda_{2}$.

(ii) If, for some $\lambda=\lambda_{0}$, the principal eigenvalue of (1.4) is zero then two possibilities arise, depending on the quantity

$$
k_{0}=\int_{\Omega} N(x) U_{0}(x) U_{0}^{*}(x) d x,
$$

where $U_{0}>0$ and $U_{0}^{*}>0$ are eigenfunctions of (2.55) and (2.56) respectively, for $\lambda=\lambda_{0}$ and $\mu=0$ :

(iia) If $k_{0} \neq 0$ then, in addition to $\lambda_{0}$ (which is obviously a principal eigenvalue), (1.5) has exactly one principal eigenvalue $\lambda_{1} \neq \lambda_{0}$, and $k_{0}\left(\lambda_{0}-\lambda_{1}\right)>0$.

(iib) If $k_{0}=0$ then $\lambda_{0}$ is the only principal eigenvalue of (1.5).

Proof. - Without loss of generality, as above, we assume that the function $M$ satisfies (2.51). For each $\lambda \in \mathbb{R}$ let $\mu=\mathcal{M}(\lambda)$ be the (unique) principal eigenvalue of

$$
\mathcal{L} U-M(x) U-\lambda N(x) U=\mu[1+|N(x)|] U \quad \text { in } \Omega, \quad U=0 \quad \text { on } \partial \Omega .
$$

Note that $\mathcal{M}$ is analytic (Proposition 2.9), and that the eigenfunction $U$ is uniquely determined if an additional condition, such as

$$
\int_{\Omega} U(x) d x=1
$$

is imposed; in this case, according to the argument in the proof of Proposition 2.9, $U$ also depends analytically on $\lambda$. Still, when using the characterization of the principal eigenvalue in Proposition 2.8 and assumptions (H.3') and (2.51), it is readily seen that $\mathcal{M}\left(\lambda_{0}\right)>0$ in case (i), and that $\mathcal{M}\left(\lambda_{0}\right)=0$ in case (ii). Then the stated result readily follows from the following properties of the function $\mathcal{M}$, which are subsequently proved below.

A. If $\mathcal{M}\left(\lambda_{0}\right)=0$ then the derivative of $\mathcal{M}$ at $\lambda=\lambda_{0}$ is given by

$$
\left\{\int_{\Omega}[1+|N(x)|] U_{0}(x) U_{0}^{*}(x) d x\right\} \mathcal{M}^{\prime}\left(\lambda_{0}\right)=-\int_{\Omega} N(x) U_{0}(x) U_{0}^{*}(x) d x .
$$

B. The function $\mathcal{M}$ is concave.

C. $\mathcal{M} \rightarrow-\infty$ as $\lambda \rightarrow \pm \infty$.

In order to prove property $A$ we just take into account that $\mathcal{M}^{\prime}\left(\lambda_{0}\right)$ is given by

$$
\begin{aligned}
& \mathcal{L} U_{0}^{\prime}-M(x) U_{0}^{\prime}-\lambda_{0} N(x) U_{0}^{\prime}=\left[N(x)+\mathcal{M}^{\prime}\left(\lambda_{0}\right)(1+|N(x)|)\right] U_{0} \text { in } \Omega, \\
& U_{0}^{\prime}=0 \text { on } \partial \Omega,
\end{aligned}
$$


as readily obtained by differentiating in (2.57), and setting $\lambda=\lambda_{0}$ and $\mathcal{M}\left(\lambda_{0}\right)=0$; here $U_{0}^{\prime}=\partial U / \partial \lambda$ at $\lambda=\lambda_{0}$ and $U_{0}=U\left(\cdot, \lambda_{0}\right)$. And (2.58) is obtained when taken into account that, since the singular, non-homogeneous problem (2.59) has a solution, its right hand side must be orthogonal to the kernel of its adjoint problem.

In order to prove property $B$ we just take into account that

$$
\lambda+\mathcal{M}(\lambda)=\Gamma(\lambda-\mathcal{M}(\lambda))
$$

for all $\lambda$, where the function $\Gamma$ is defined as follows. For each $\mu \in \mathbb{R}, \Gamma(\mu)$ is the (unique) principal eigenvalue of

$$
\mathcal{L} U-M(x) U+\mu N_{-}(x) U=\Gamma(\mu) N_{+}(x) U \quad \text { in } \Omega, \quad U=0 \quad \text { on } \partial \Omega,
$$

where $N_{ \pm}=N^{ \pm}+1 / 2$ and $N^{ \pm}$are the positive/negative parts of $N$ (i.e., $N^{ \pm} \geqslant 0$ and $N=N^{+}-N^{-}$). And since $\Gamma$ is analytic, (strictly) increasing and concave (Proposition 2.9), the statement in property $B$ readily follows because $\left(1+\Gamma^{\prime}\right) \mathcal{M}^{\prime \prime}=$ $\left(1-\mathcal{M}^{\prime}\right)^{2} \Gamma^{\prime \prime}$, as readily obtained by differentiating twice in (2.60).

Finally, in order to prove property $C$, we consider two non-void, open balls, $\mathcal{B}_{ \pm}$, such that $\overline{\mathcal{B}}_{ \pm} \subset \Omega$ and $N^{ \pm}>0$ in $\overline{\mathcal{B}}_{ \pm}$, and define the strictly positive constants $k_{ \pm}$and $k^{ \pm}$ as the minimum and the maximum of $N^{ \pm}$in $\mathcal{B}_{ \pm}$, respectively. If $\lambda_{0}^{ \pm}$are the principal eigenvalues of

$$
\mathcal{L} U-M(x) U=\lambda_{0}^{ \pm} U \quad \text { in } \mathcal{B}_{ \pm}, \quad U=0 \quad \text { on } \partial \mathcal{B}_{ \pm},
$$

and $\pm \lambda>\lambda_{0}^{ \pm} / k_{ \pm}$, then $\left(1+k_{ \pm}\right) \mathcal{M}(\lambda) \pm \lambda k_{ \pm} \leqslant \lambda_{0}^{ \pm}$, as readily obtained upon application of the generalized maximum principle to (2.62) (recall the definition of the function $\mathcal{M}$, through (2.57)). These two inequalities yield the result, and the proof is complete.

\section{Differentiability of some singular nonlinear problems}

Let us now consider the linearization (in fact, the differentiability) of the semilinear problem (1.1) around a solution $u \in C^{2}(\Omega) \cap C^{1}(\bar{\Omega})$ such that

$$
u>0 \quad \text { in } \Omega, \quad \partial u / \partial v<0 \quad \text { on } \partial \Omega .
$$

If $\alpha>-1 / n$ then we can treat the problem (1.1) in differential form and work in the space $W_{p}^{2}(\Omega) \cap W_{1,0}^{p}(\Omega)$, with $p>n$, which is compactly imbedded into $C_{0}^{1}(\bar{\Omega})$; but this is not convenient for the general case treated in this paper, $\alpha>-1$ (see Remark 2.4 above). Instead we shall re-write (1.1) in integral form, as

$$
\mathcal{F}(u) \equiv u-G(f(\cdot, u))=0
$$

where $G: C_{0}^{1}(\bar{\Omega}) \rightarrow C_{0}^{1}(\bar{\Omega})$ is the Green operator of

$$
\mathcal{L} U=V \quad \text { in } \Omega, \quad U=0 \quad \text { on } \partial \Omega,
$$


(defined as $G(V)=U$ ). Note that, according to Proposition 2.3, (a) $G$ is bounded and can be extended, as a bounded operator, to $C_{0}^{1, \delta}(\bar{\Omega})$ for all $\delta$ such that $0<\delta<\delta_{0}=$ $\min \{\gamma, \alpha+1\}$; and (b) $u \in C^{2}(\Omega) \cap C^{1}(\bar{\Omega})$ satisfies (1.1) if and only if $u \in C_{0}^{1}(\bar{\Omega})$ satisfies (3.2).

THEOREM 3.1. - Under the assumptions (H.1)-(H.3), the operator $\mathcal{F}: C_{0}^{1}(\bar{\Omega}) \rightarrow$ $C_{0}^{1}(\bar{\Omega})$ defined in (3.2) is of class $C^{m}$ in the positive cone of $C_{0}^{1}(\bar{\Omega})$ (that is, the set of those functions of $C_{0}^{1}(\bar{\Omega})$ that satisfy $(3.1)$ ), where $m \geqslant 1$ is as defined in assumption (H.3) and the linear operator $\mathcal{F}^{\prime}(u): C_{0}^{1}(\bar{\Omega}) \rightarrow C_{0}^{1}(\bar{\Omega})$ is given by

$$
\mathcal{F}^{\prime}(u) v=v-G\left(f_{u}(\cdot, u) v\right) .
$$

If $m>1$ and $1<j \leqslant m$ then the $j$-linear operator, $\partial^{j} \mathcal{F}(u) / \partial u^{j} \equiv \mathcal{F}^{(j)}(u):\left[C_{0}^{1}(\bar{\Omega})\right]^{j}$ $\rightarrow C_{0}^{1}(\bar{\Omega})$, is given by

$$
\mathcal{F}^{(j)}(u)\left(v_{1}, \ldots, v_{j}\right)=-G\left(\left(\partial^{j} f(\cdot, u) / \partial u^{j}\right) v_{1} \cdots v_{j}\right) .
$$

Proof. - The operator $\mathcal{F}$ can be written as

$$
\mathcal{F}=I-\mathcal{F}_{1}, \quad \text { with } I=\text { identity and } \mathcal{F}_{1}=G(f(\cdot, u)) .
$$

Since $I$ is linear and bounded, it is of class $C^{\infty}$, with its first derivative equal to $I$ and its higher order derivatives equal to zero. Thus we only need to prove that $(a)$ for $j=1, \ldots, m$, the $j$ th derivative of $\mathcal{F}_{1}$ exists and is given by

$$
\mathcal{F}_{1}^{(j)}(u)\left(v_{1}, \ldots, v_{j}\right)=G\left(\left(\partial^{j} f(\cdot, u) / \partial u^{j}\right) v_{1} \cdots v_{j}\right),
$$

and (b) the $m$ th derivative of $\mathcal{F}_{1}$ is continuous.

Let us first prove (a) by an induction argument. In order to prove (a) for $j=1$ we first consider a function $N \in C^{2}(\Omega)$ such that $N \geqslant 1$ in $\Omega$ and $N(x)=d(x)^{\alpha-1}$ for all $x \in \Omega_{1}$ (with $d(x)$ and $\Omega_{1}$ as defined in assumption (H.1)). Note that

$$
d(x)^{\alpha-1} / N \text { and }\left|\partial N / \partial x_{k}\right| d(x)^{2-\alpha} \quad \text { are uniformly bounded in } \Omega,
$$

for $k=1, \ldots, n$. Now, take any function $u$ of the positive cone of $C_{0}^{1}(\bar{\Omega})$. If $v \in C_{0}^{1}(\bar{\Omega})$ is such that $\|v\|_{C_{0}^{1}(\bar{\Omega})}$ is sufficiently small then

$$
\begin{gathered}
0<k_{1} d(x)<u+\theta v<k_{2} d(x), \\
\quad \text { for all } x \in \bar{\Omega} \text { and all } \theta \in[0,1],
\end{gathered}
$$

where the constants $k_{1}$ and $k_{2}$ are independent of $v$. Thus, according to assumption (H.3) and property (3.8), the function

$$
W \equiv\left[f(\cdot, u+v)-f(\cdot, u)-f_{u}(\cdot, u) v\right] / N,
$$

is such that

$$
|W|=\left|f_{u u}(x, u+\theta(x) v)\right| v^{2} / N \leqslant K_{1}\|v\|_{C_{0}^{1}(\bar{\Omega})}^{2}
$$




$$
\begin{aligned}
\left|W_{x_{k}}\right| \leqslant & \mid f_{u x_{k}}(x, u+\theta(x) v)-f_{u x_{k}}(x, u)+\left[f_{u u}(x, u+\theta(x) v)\right. \\
& \left.-f_{u u}(x, u)\right] u_{x_{k}}|| v|/ N+| f_{u u}(x, u+\theta(x) v)|| v v_{x_{k}}|/ N+| W N_{x_{k}} \mid / N^{2} \\
\leqslant & K_{2}\|v\|_{C_{0}^{1}(\bar{\Omega})} \varepsilon\left(\|v\|_{C_{0}^{1}(\bar{\Omega})}\right),
\end{aligned}
$$

for all $k=1, \ldots, n$ and all $v \in C_{0}^{1}(\bar{\Omega})$ such that $\|v\|_{C_{0}^{1}(\bar{\Omega})} \leqslant\|u\|_{C_{0}^{1}(\bar{\Omega})}$. Here $\theta$ and $\varepsilon$ stand for functions of the type $\theta: \Omega \rightarrow[0,1]$ and $\varepsilon: \mathbb{R} \rightarrow \mathbb{R}$, with $\varepsilon(z) \rightarrow 0$ as $z \rightarrow 0$, and the constants $K_{1}$ and $K_{2}$ are independent of $v$. Thus $\|W\|_{C_{0}^{1}(\bar{\Omega})} /\|v\|_{C_{0}^{1}(\bar{\Omega})} \leqslant$ $\left(K_{1}+K_{2}\right) \varepsilon\left(\|v\|_{C_{0}^{1}(\bar{\Omega})}\right)$ and we only need to take into account the definitions (3.6) and (3.10), and the result in Proposition 2.3 to subsequently obtain

$$
\begin{aligned}
\left\|\mathcal{F}_{1}(u+v)-\mathcal{F}_{1}(u)-G\left(f_{u}(\cdot, u) v\right)\right\|_{C_{0}^{1}(\bar{\Omega})} & =\|G(N W)\|_{C_{0}^{1}(\bar{\Omega})} \\
& \leqslant K\|v\|_{C_{0}^{1}(\bar{\Omega})} \varepsilon\left(\|v\|_{C_{0}^{1}(\bar{\Omega})}\right)
\end{aligned}
$$

for all $v \in C_{0}^{1}(\bar{\Omega})$ such that $\|v\|_{C_{0}^{1}(\bar{\Omega})} \leqslant\|u\|_{C_{0}^{1}(\bar{\Omega})}$ and some constant $K$ that is independent of $v$. This estimate implies that $\mathcal{F}_{1}^{\prime}(u)$ exists and is given by (3.7). Thus property (a) above holds for $j=1$ and the first step of the induction argument is complete.

Let us now assume that property (a) holds for the $j$ th derivative of $\mathcal{F}_{1}$ and prove that it also holds for its $(j+1)$ th derivative. To this end, we take $v_{1}, \ldots, v_{j+1} \in C_{0}^{1}(\bar{\Omega})$ such that $\left\|v_{j+1}\right\|_{C_{0}^{1}(\bar{\Omega})} \leqslant\|u\|_{C_{0}^{1}(\bar{\Omega})}$. Then (3.9) holds with $v=v_{j+1}$ and, as above, according to assumption (H.3), the function

$$
\begin{aligned}
W_{1} \equiv & \left\{\partial^{j} f\left(\cdot, u+v_{j+1}\right) / \partial u^{j}-\partial^{j} f(\cdot, u) / \partial u^{j}\right. \\
& \left.-\left[\partial^{j+1} f(\cdot, u) / \partial u^{j+1}\right] v_{j+1}\right\} v_{1} \cdots v_{j} / N
\end{aligned}
$$

is seen to be such that

$$
\left\|W_{1}\right\|_{C_{0}^{1}(\bar{\Omega})} \leqslant K_{3}\left\|v_{1}\right\|_{C_{0}^{1}(\bar{\Omega})} \cdots\left\|v_{j}\right\|_{C_{0}^{1}(\bar{\Omega})}\left\|v_{j+1}\right\|_{C_{0}^{1}(\bar{\Omega})} \varepsilon\left(\left\|v_{j+1}\right\|_{C_{0}^{1}(\bar{\Omega})}\right),
$$

with $K_{3}$ independent of $v_{1}, \ldots, v_{j+1}$ and $\varepsilon$ as above. Thus, as above, we only need to take into account our assumption that $\mathcal{F}_{1}^{(j)}(u)$ is given by (3.7), the definition (3.14) and the result in Proposition 2.3 to subsequently obtain

$$
\begin{aligned}
& \left\|\left[\mathcal{F}_{1}^{(j)}\left(u+v_{j+1}\right)-\mathcal{F}_{1}^{(j)}(u)\right]\left(v_{1}, \ldots, v_{j}\right)-G\left(\left(\partial^{j+1} f(\cdot, u) / \partial u^{j}\right) v_{1} \cdots v_{j}\right)\right\|_{C_{0}^{1}(\bar{\Omega})} \\
& \quad=\left\|G\left(N W_{1}\right)\right\|_{C_{0}^{1}(\bar{\Omega})} \\
& \quad \leqslant K\left\|v_{1}\right\|_{C_{0}^{1}(\bar{\Omega})} \cdots\left\|v_{j}\right\|_{C_{0}^{1}(\bar{\Omega})}\left\|v_{j+1}\right\|_{C_{0}^{1}(\bar{\Omega})} \varepsilon\left(\left\|v_{j+1}\right\|_{C_{0}^{1}(\bar{\Omega})}\right),
\end{aligned}
$$

with $K$ independent of $v_{1}, \ldots, v_{j+1}$. This estimate shows that $\mathcal{F}_{1}^{(j+1)}(u)$ exists and is given by (3.7). Thus the induction argument is complete and property (a) above holds.

Finally, the same argument that led above to (3.15) readily shows that

$$
\left\|\left[\mathcal{F}_{1}^{(m)}\left(u+v_{j+1}\right)-\mathcal{F}_{1}^{(m)}(u)\right]\left(v_{1}, \ldots, v_{j}\right)\right\|_{C_{0}^{1}(\bar{\Omega})} \leqslant K\left\|v_{1}\right\|_{C_{0}^{1}(\bar{\Omega})} \cdots\left\|v_{j}\right\|_{C_{0}^{1}(\bar{\Omega})}\left\|v_{j+1}\right\|_{C_{0}^{1}(\bar{\Omega})},
$$

with $K$ independent of $v_{1}, \ldots, v_{j+1}$. According to this estimate, $\mathcal{F}_{1}^{(m)}$ is continuous. Thus property (b) above also holds, and the proof is complete. 
If the function $f$ depends also on a parameter, then the same argument in the proof of Theorem 3.1 readily yields the following

COROLLARY 3.2. - In addition to the assumptions of Theorem 3.1, let us assume that the function $f$ depends on a parameter $\lambda \in \mathbb{R}$ and that, for all $\lambda \in \mathbb{R}$ and all $l=1, \ldots, r$, the function $\partial^{l} f / \partial \lambda^{l}$ satisfies assumption (H.3). If the Green operator $G$ is defined as above, right after (3.2), then the operator $\mathcal{F}: \Omega \times C_{0}^{1}(\bar{\Omega}) \times \mathbb{R}$, defined as

$$
\mathcal{F}(u, \lambda) \equiv u-G(f(\cdot, u, \lambda)),
$$

is such that, for all $j=0, \ldots, m$ and all $l=1, \ldots, r$, the derivative $\partial^{j+l} \mathcal{F} / \partial u^{j} \partial \lambda^{l}$ exists and is continuous whenever $u$ is in the positive cone of $C_{0}^{1}(\bar{\Omega})$ and $\lambda \in \mathbb{R}$. Also, the $j$ linear operator $\partial^{j+l} \mathcal{F}(u, \lambda) / \partial u^{j} \lambda^{l}:\left[C_{0}^{1}(\bar{\Omega})\right]^{j} \rightarrow C_{0}^{1}(\bar{\Omega})$ is given by

$$
\left[\partial^{j+l} \mathcal{F}(u, \lambda) / \partial u^{j} \lambda^{l}\right]\left(v_{1}, \ldots, v_{j}\right)=-G\left(\left(\partial^{j+l} f(\cdot, u, \lambda) / \partial u^{j} \partial \lambda^{l}\right) v_{1} \cdots v_{j}\right) .
$$

Note that this corollary provides the ingredient to apply implicit-function-like theorems to the problem (1.1)-(1.2) when the nonlinearity $f$ is allowed to depend also on a parameter.

\section{Applications}

The results obtained in Sections 2 and 3 and the strong maximum principle in Appendix B provide the basic ingredients to systematically apply the standard tools mentioned at the beginning of the paper to the analysis of singular equations of the form (1.1)-(1.2), under the assumptions (H.1)-(H.3).

\subsection{Construction of solutions of (1.1)-(1.2) via sub and supersolutions}

Monotone methods can be applied, in quite the same manner as is the regular case, to construct minimal and maximal solutions of (1.1)-(1.2) in the positive cone of $C_{0}^{1}(\bar{\Omega})$, as seen in the proof of the following

THEOREM 4.1. - Under the assumptions (H.1)-(H.3), let us assume that (1.1)-(1.2) has a sub-solution $u_{0}$ and a super-solution $u^{0}$ such that $u_{0}, u^{0} \in C^{2}(\Omega) \cap C_{0}^{1, \delta}(\bar{\Omega})$ for some $\delta>0$ and

$$
0<k d(x)<u_{0}(x) \leqslant u^{0}(x) \text { for all } x \in \Omega .
$$

Then (1.1)-(1.2) possesses a minimal and a maximal solution in the interval $\left[u_{0}, u^{0}\right]$, $u_{*}$ and $u^{*}$, which are such that $u_{*}, u^{*} \in C^{2}(\Omega) \cap C_{0}^{1, \delta}(\bar{\Omega})$ whenever $0<\delta<\delta_{0}=$ $\min \{\gamma, \alpha+1\}$ and $u_{0} \leqslant u_{*} \leqslant u^{*} \leqslant u^{0}$ in $\Omega$. Also, $u_{*}$ (resp., $\left.u^{*}\right)$ is the $C_{0}^{1, \delta}(\bar{\Omega})$-limit from below (resp., from above) of a monotone sequence of sub-solutions (resp., supersolutions) of (1.1)-(1.2).

Proof. - According to assumption (H.3), $\left|f_{u}(x, u(x))\right| d(x)^{1-\alpha}$ is bounded in $\Omega$ if $u: \Omega \rightarrow \mathbb{R}$ is such that $u_{0} \leqslant u \leqslant u^{0}$ in $\Omega$. Thus a function $M_{1} \in C^{1}(\Omega)$ exists that satisfies (H.3') and is such that, for some constants $k>0$ and $\beta \in] 1-\alpha, 2[$, 


$$
\begin{aligned}
& M_{1}>1 \quad \text { in } \Omega \backslash \Omega_{1}, \quad M_{1}(x)=k d(x)^{-\beta} \quad \text { for all } x \in \Omega_{1}, \\
& \text { and } f(\cdot, u) / u+\left|f_{u}(\cdot, u)\right|<M_{1} \quad \text { in } \Omega \text { if } u_{0} \leqslant u \leqslant u^{0} \text { in } \Omega,
\end{aligned}
$$

where $\Omega_{1}$ is as defined in assumption (H.1). Then the mean value theorem and assumption (H.3) imply that the functions $\varphi, \psi: \Omega \rightarrow \mathbb{R}$, defined as

$$
\varphi(x, u) \equiv M_{1}(x) u+f(x, u) \equiv M_{1}(x) \psi(x, u),
$$

are such that

$$
\begin{aligned}
& 0<\varphi(\cdot, u)<\varphi(\cdot, v) \quad \text { in } \Omega, \quad \psi(\cdot, u) \in C_{0}^{1}(\bar{\Omega}), \\
& \|\psi(\cdot, u)\|_{C^{1}(\bar{\Omega})} \leqslant K_{1}\|u\|_{C^{1}(\bar{\Omega})} \quad \text { and } \\
& \|\psi(\cdot, v)-\psi(\cdot, u)\|_{C^{1}(\bar{\Omega})}<K_{2}\|v-u\|_{C^{1}(\bar{\Omega})} \quad \text { in } \Omega, \\
& \text { whenever } u_{0} \leqslant u<v \leqslant u^{0} \quad \text { in } \Omega,
\end{aligned}
$$

for some constants $K_{1}$ and $K_{2}$ that are independent of $u$ and $v$.

Now we consider the sequences $\left\{u_{m}\right\},\left\{u^{m}\right\}$, defined inductively by

$$
\begin{array}{llll}
\mathcal{L} u_{m}+M_{1}(x) u_{m}=\varphi\left(x, u_{m-1}(x)\right) & \text { in } \Omega, & u_{m}=0 & \text { on } \partial \Omega, \\
\mathcal{L} u^{m}+M_{1}(x) u^{m}=\varphi\left(x, u^{m-1}(x)\right) & \text { in } \Omega, & u^{m}=0 & \text { on } \partial \Omega,
\end{array}
$$

for $m>0$, with $u_{0}$ and $u^{0}$ as above. Since $\varphi$ and $\psi=\varphi / M_{1}$ satisfy (4.5), we only need to apply the maximum principle in Appendix B and Proposition 2.3 to obtain inductively that $\left\{u_{m}\right\},\left\{u^{m}\right\} \subset C^{2}(\Omega) \cap C^{1, \delta}(\bar{\Omega})$ whenever $m \geqslant 1$ and $0<\delta<\delta_{0}=\min \{\gamma, \alpha+1\}$, and, as in the regular case, if $u$ is a solution of (1.1)-(1.2) such that $u_{0} \leqslant u \leqslant u^{0}$ in $\Omega$, then

$$
u_{0} \leqslant u_{m-1} \leqslant u_{m} \leqslant u \leqslant u^{m} \leqslant u^{m-1} \leqslant u^{0} \quad \text { in } \Omega \text { for all } m>1 .
$$

Hence the stated result follows if we prove that

$$
\left\{u_{m}\right\} \text { and }\left\{u^{m}\right\} \text { converge in } C^{1, \delta}(\bar{\Omega}) \text { whenever } 0<\delta<\delta_{0}=\min \{\gamma, \alpha+1\}
$$

(then, according to standard, local, elliptic estimates, the limits must be in $C^{2}(\Omega)$ ).

Now, in order to obtain (4.9) for the monotone, bounded sequence $\left\{u_{m}\right\}$ (the other sequence is treated similarly) we first observe that, by the dominated convergence theorem, it converges in $L_{q}(\Omega)$ for all $q>1$. Also, according to Proposition 2.3 and properties (4.5), if $p>m>1$ and $\delta$ is as above, then

$$
\left\|u_{p}-u_{m}\right\|_{C^{1, \delta}(\bar{\Omega})}<K\left\|u_{p-1}-u_{m-1}\right\|_{C^{1}(\bar{\Omega})},
$$

with the constant $K$ independent of $m$ and $p$. In addition we have the interpolation inequality $\|u\|_{C^{1}(\bar{\Omega})}<\varepsilon\|u\|_{C^{1, \delta}(\bar{\Omega})}+C_{\varepsilon, q}\|u\|_{L_{q}(\Omega)}$, which holds for all $\varepsilon>0$ and all $q>n+2$ (see [2] and [32, p. 80]). Thus, if $q>n+2$ is kept fixed, this inequality (with $\varepsilon>0$ appropriate) and (4.10) readily yield

$$
\begin{aligned}
& \left\|u_{p}-u_{m}\right\|_{C^{1}(\bar{\Omega})}<(1 / 4)\left\|u_{p-1}-u_{m-1}\right\|_{C^{1}(\bar{\Omega})}+K_{1}\left\|u_{p}-u_{m}\right\|_{L_{q}(\Omega)} \\
& <\left(\left\|u_{p}-u_{m}\right\|_{C^{1}(\bar{\Omega})}+\left\|u_{p}-u_{p-1}\right\|_{C^{1}(\bar{\Omega})}+\left\|u_{m}-u_{m-1}\right\|_{C^{1}(\bar{\Omega})}\right) / 4 \\
& \quad+K_{1}\left(\left\|u_{p}-u_{m}\right\|_{L_{q}(\Omega)}\right),
\end{aligned}
$$


where $K_{1}$ is independent of $m$ and $p$. Since $\left\{u_{m}\right\}$ converges in $L_{q}$, the first inequality in (4.11) (with $p=m+1$ ) implies that $\left\|u_{m+1}-u_{m}\right\|_{C^{1}(\bar{\Omega})} \rightarrow 0$ as $m \rightarrow \infty$. And, since $\left\{u_{m}\right\}$ is a Cauchy sequence in $L_{q},(4.11)$ and (4.10) subsequently imply that $\left\{u_{m}\right\}$ is a Cauchy sequence in $C^{1}(\bar{\Omega})$ and in $C^{1, \delta}(\bar{\Omega})$. Thus $\left\{u_{m}\right\}$ satisfies (4.9) and the proof is complete.

The requirement that $u_{0}>k d(x)>0$ in $\Omega$ in Theorem 4.1 is often too strong in applications. For instance, if the unique solution of

$$
\mathcal{L} U=M(x) \quad \text { in } \Omega, \quad U=0 \quad \text { on } \partial \Omega,
$$

is strictly positive in $\Omega$ then according to Lemma 4.3 below $u_{0}=\left[\left(1-\alpha_{1}\right) U\right]^{1 /\left(1-\alpha_{1}\right)}$ is a strict sub-solution of

$$
\mathcal{L} u=M(x) u^{\alpha_{1}} \quad \text { in } \Omega, \quad u=0 \quad \text { on } \partial \Omega .
$$

But this sub-solution does not satisfy the above-mentioned requirement if $U \in C_{0}^{1}(\bar{\Omega})$. In order to extend the applicability of Theorem 4.1 to situations like this one, in the following lemma we prove that the above-mentioned requirement in Theorem 4.1 can be weakened.

LEMMA 4.2. - Under the assumptions of Theorem 4.1, let the function $f$ (satisfy (H.3) and ) be such that $f(x, u)>-K_{1} d(x)^{\alpha_{2}} u^{\alpha_{1}}$ and $|f(x, u)|+\left|f_{u}(x, u)\right| u<$ $K_{2} d(x)^{\alpha_{4}} u^{\alpha_{3}}$ for all $\left.(x, u) \in \Omega \times\right] 0, \infty\left[\right.$, with $K_{1}>0, K_{2}>0,\left|\alpha_{1}+\alpha_{2}\right|<1$ and $\left|\alpha_{3}+\alpha_{4}\right|<1$, and let $\tilde{u}_{0} \in C^{2}(\Omega) \cap C_{0}^{1, \delta}(\bar{\Omega})$, for some $\delta>0$, be a sub-solution of (1.1)(1.2) such that $\tilde{u}_{0}>\tilde{k} d(x)^{p}$ a.e. in $\Omega$, with $\tilde{k}>0$ and $p>1$. In addition, let us assume that either (a) $\alpha_{1} \geqslant 1$, or (b) $0<\left(1-\alpha_{1}\right) /\left(2+\alpha_{2}\right)<\alpha_{1}<1$ and $\alpha_{4}+\alpha_{3} / \alpha_{1}>-1$, or (c) $\alpha_{1}<\left(1-\alpha_{1}\right) /\left(2+\alpha_{2}\right)<1 / p$ and $p \alpha_{3}+\alpha_{4}>-1$. Then there is a sub-solution of (1.1)-(1.2), $u_{0} \in C^{2}(\Omega) \cap C_{0}^{1, \delta}(\bar{\Omega})(\delta>0)$, such that (i) $u_{0}>k d(x)$ in $\Omega$ for some $k>0$, and (ii) $u_{0}<u$ in $\Omega$ whenever $u \in C^{2}(\Omega) \cap C_{0}^{1, \delta}(\bar{\Omega})(\delta>0)$ is a solution of (1.1)-(1.2) such that $\tilde{u}_{0}<u$ and $0<k_{1} d(x)<u$ in $\Omega$ for some $k_{1}>0$.

Proof. - Under the assumptions above, we can choose a constant $r$ and a function $\rho:[0, \infty[\rightarrow \mathbb{R}$ such that

$$
1<r<p, \quad r \alpha_{3}+\alpha_{4}>r-2 \quad \text { and } \quad \rho(\varepsilon)=\varepsilon^{1 /[2(p-r)]}
$$

in case (a) above, and

$$
\begin{aligned}
& 1<r, \quad\left[2+\alpha_{2}+r\left(\alpha_{1}-1\right)\right] /\left(1-\alpha_{1}\right)>\max \{0, p-r\}, \quad r \alpha_{3}+\alpha_{4}>r-2 \\
& \text { and } \rho(\varepsilon)=\varepsilon^{\left(1-\alpha_{1}\right) /\left[2+\alpha_{2}+r\left(\alpha_{1}-1\right)\right]}
\end{aligned}
$$

in cases (b) and (c). Also we consider the subdomain $\Omega_{\varepsilon}$ and the function $u_{\varepsilon}: \Omega_{\varepsilon} \rightarrow \mathbb{R}$ defined as

$$
\Omega_{\varepsilon}=\{x \in \Omega: d(x)<\rho(\varepsilon)\} \quad \text { and } \quad u_{\varepsilon}(x)=\varepsilon d(x)^{r},
$$

which are seen to be such that, in the three cases considered above, (i) $u_{\varepsilon} \in C^{2}\left(\Omega_{\varepsilon}\right) \cap$ $C_{0}^{1}\left(\bar{\Omega}_{\varepsilon}\right)$, (ii) $u_{\varepsilon}<u_{0}$ in $\partial \Omega \backslash \partial \Omega_{\varepsilon}$ and (iii) $u_{\varepsilon}$ is a sub-solution of (1.1)-(1.2) in $\Omega_{\varepsilon}$, 
whenever $\varepsilon>0$ is sufficiently small. The first property is obvious, and the second and third ones follow from (4.14)-(4.16) and the stated assumptions on $u_{0}$ and $f$; the proof of the third property is somewhat tedious but straightforward. As a consequence of these three properties, the function $\tilde{u}_{\varepsilon}$, defined as

$$
\tilde{u}_{\varepsilon}(x)=\tilde{u}_{0}(x) \quad \text { if } x \in \Omega \backslash \Omega_{\varepsilon}, \quad \tilde{u}_{\varepsilon}=\max \left\{u_{\varepsilon}, \tilde{u}_{0}(x)\right\} \quad \text { if } x \in \Omega_{\varepsilon},
$$

is such that $\tilde{u}_{\varepsilon} \leqslant \tilde{u}_{0}$ in $\Omega$ and $\tilde{u}_{\varepsilon}$ is a sub-solution of (1.1)-(1.2) whenever $\varepsilon>0$ is sufficiently small. Also, $\varepsilon>0$ can be chosen such that $u_{\varepsilon}<u$ if $\mathrm{u}$ is as in the statement of the lemma. For that value of $\varepsilon$ we choose a function $M_{1} \in C^{1}(\Omega)$ that satisfies (4.2) and

$$
|f(\cdot, u)| / u+\left|f_{u}(\cdot, u)\right|<M_{1} \quad \text { in } \Omega \text { if } u_{\varepsilon} \leqslant u \leqslant u^{0} \text { in } \Omega,
$$

with $r-\left(r \alpha_{3}+\alpha_{4}\right)<\beta<2$ and calculate $u_{0}$ as the unique solution of

$$
\mathcal{L} u_{0}+M_{1}(x) u_{0}=M_{1}(x) u_{\varepsilon}+f\left(x, u_{\varepsilon}(x)\right) \quad \text { in } \Omega, \quad u_{0}=0 \quad \text { on } \partial \Omega .
$$

Finally, by the argument in the proof of Theorem 4.1 (write the right hand side of (4.18) as $M_{1}(x) \psi\left(x, u_{\varepsilon}(x)\right)$ and check that the function $x \rightarrow \psi\left(x, u_{\varepsilon}(x)\right)$ is in $\left.C_{0}^{0,1}(\bar{\Omega})\right)$, $u_{0} \in C^{2}(\Omega) \cap C_{0}^{1, \delta}(\bar{\Omega})$ (with $\delta>0$ ) is a sub-solution of (1.1)-(1.2) that satisfies the properties (i) and (ii) in the statement of the lemma. Thus the proof is complete.

The following result is of independent interest to obtain sub-solutions of semilinear equations with a monotone nonlinearity. It is based on the generalization to elliptic operators in general form of an argument already used by Spruck [44] to treat the operator $\mathcal{L}=-\Delta$; see also [10] where this argument was credited to Nirenberg. In the present context, this argument is based on the fact that if the functions $u$ and $U\left(\in C^{2}(\Omega)\right)$ are related by

$$
u(x)=\int_{0}^{U(x)} d t / f(t),
$$

where $f \in C(] 0, \infty[)$ is strictly positive and such that the integral above is convergent, then we have

$$
\mathcal{L} U=\frac{\mathcal{L} u}{f(u)}+\frac{f^{\prime}(u)}{f(u)^{2}} \sum a_{i j}(x) \frac{\partial u}{\partial x_{i}} \frac{\partial u}{\partial x_{j}} \quad \text { in } \Omega .
$$

Note that both (4.19) and (4.20) make sense whenever the integral above is convergent and $u>0$ in $\Omega$, irrespectively on how fast $u(x)$ decays as $x$ approaches $\partial \Omega$.

LEMmA 4.3. - Let $f \in C^{1}(] 0, \infty[)$ be a strictly increasing, positive function such that $\int_{0}^{\delta} d t / f(t)<\infty$ for some $\delta>0$ and let $M \in C(\Omega)$ and $U \in C^{2}(\Omega)$ be such that

$$
\mathcal{L} U \leqslant M, \quad U>0 \quad \text { in } \Omega,
$$

where the domain $\Omega$ and the operator $\mathcal{L}$ are as in assumptions (H.1) and (H.2). Then the function $u \in C^{2}(\Omega)$ defined by (4.19) satisfies

$$
\mathcal{L} u<M(x) f(u) \quad \text { in } \Omega .
$$


Proof. - We only need to use (4.20) and take into account that $f$ is positive and strictly increasing and that $\mathcal{L}$ is strongly elliptic to obtain

$$
\begin{aligned}
0 & =(\mathcal{L} U-M) f(u)=\mathcal{L} u+\frac{f^{\prime}(u)}{f(u)} \sum a_{i j}(x) \frac{\partial u}{\partial x_{i}} \frac{\partial u}{\partial x_{j}}-M(x) f(u) \\
& >\mathcal{L} u-M(x) f(u) .
\end{aligned}
$$

Now we have the ingredients to obtain existence and uniqueness of positive solutions to (4.13).

THEOREM 4.4. - Let $\Omega$ and $\mathcal{L}$ satisfy assumptions (H.1)-(H.2), let $\alpha_{1}$ be such that $0<\alpha_{1}<1$ and let $M \in C^{1}(\Omega)$ be such that (i) $|M(x)|<K_{1} d(x)^{\alpha_{2}}$ for some $K_{1}>0$ and some $\alpha_{2}$ such that $-1<\alpha_{2}<1-\alpha_{1}$, and (ii) the unique solution of (4.12) satisfies $U>0$ in $\Omega$ and $\partial U / \partial v<0$ on $\partial \Omega$. Then the problem (4.13) has a unique strictly positive solution.

Proof. - Note that (4.12) has a unique solution $U \in C^{2}(\Omega) \cap C^{1, \delta}(\bar{\Omega})(\delta>0)$, as obtained from, e.g., Proposition 2.3 above, after re-writing the right hand side of (4.12) as $[M(x) / v(x)] v(x)$ for some function $v \in C_{0}^{1}(\Omega)$. Now we may apply Lemma 4.3 above, with $f(u)=u^{\alpha_{1}}$, to obtain that $\tilde{u}_{0}=\left[\left(1-\alpha_{1}\right) U\right]^{1 /\left(1-\alpha_{1}\right)}$ is a strict sub-solution of (4.13). This sub-solution satisfies the assumptions in Lemma 4.2 and thus (4.13) possesses a positive sub-solution satisfying the requirements of Theorem 4.1. Also, if $\psi \in C^{2}(\Omega) \cap$ $C^{1, \delta}(\bar{\Omega})(\delta>0)$ is the unique solution of $\mathcal{L} \psi=|M(x)|$ in $\Omega, \psi=0$ on $\partial \Omega$, then $\psi>0$ in $\Omega$ and $\partial \psi / \partial v<0$ on $\partial \Omega$ (Theorem B.2 in Appendix B), and for sufficiently large $C>0$ the function $u^{0}=C \psi$ is a strict super-solution of (4.13) that satisfies (4.1). Thus the existence of a positive solution to (4.13) follows from Theorem 4.1.

Uniqueness of positive solutions is proved by the argument in the proof of Lemma 4.3 above. Assume for contradiction that (4.13) has two different positive solutions, $u_{1}$ and $u_{2}$. Then the function $w$ defined as $w=v_{2}-v_{1}$, where $v_{j}=\int_{0}^{u_{j}} d t / f(t)$ for $j=1,2$ and $f(t)=t^{\alpha_{1}}$, is non-zero. But using (4.20) and applying the mean value theorem we obtain

$$
\begin{aligned}
\mathcal{L} w & -f^{\prime}\left(u_{1}\right) \sum a_{i j}(x) \frac{\partial\left(u_{1}+u_{2}\right)}{\partial x_{i}} \frac{\partial w}{\partial x_{j}} \\
- & f^{\prime \prime}\left(u_{1}+\theta_{1}(x)\left(u_{2}-u_{1}\right)\right) f\left(u_{1}+\theta_{2}(x)\left(u_{2}-u_{1}\right)\right) \\
& \times \sum a_{i j}(x) \frac{\partial v_{2}}{\partial x_{i}} \frac{\partial v_{2}}{\partial x_{j}} w=0,
\end{aligned}
$$

where we have taken into account that $u_{1}$ and $u_{2}$ satisfy (4.13). Since $f(u)=u^{\alpha_{1}}>0$, $f^{\prime \prime}(u)=\alpha_{1}\left(\alpha_{1}-1\right) u^{\alpha_{1}-2}<0$ and the operator $\mathcal{L}$ is elliptic, the coefficient of $w$ is positive in $\Omega$ and Theorem B.1 in Appendix B implies that $w=0$ in $\Omega$, which is in contradiction with the assumption above. Thus the proof is complete.

Remarks 4.5. - Two remarks about the result above are now in order.

A. From the proof it is clear that the result in Theorem 4.4 stands when the right hand side of (4.13) is replaced by $M(x) f(u)$, with $M$ as above and $f \in C([0, \infty[) \cap$ $C^{1}(] 0, \infty\left[\right.$ such that $f(0)=0, f>0, f^{\prime}>0$ and $f^{\prime \prime}<0$ in $] 0, \infty\left[, f(u)<K u^{\alpha_{1}}\right.$ in a neighborhood of $u=0$, with $K>0$ and $\alpha_{1}$ as in the statement of Theorem 4.4 and $\int_{0}^{\delta} d u / f(u)<\infty$ for some $\delta>0$. 
B. The problem (4.13) has received a considerable attention in the literature. For the particular case $\mathcal{L}=-\Delta$ and $M(x) \equiv 1$, existence of solutions has been analyzed using sub and supersolutions [4,37,43], variational arguments [11] and both [20]; see also [44, 11,10] for uniqueness. Some results for the case $0<M<\infty$ in $\bar{\Omega}$ and $\mathcal{L}$ in general form had already been given by Amann [3]. The case $\mathcal{L}=-\Delta$ with $M$ smooth and bounded but exhibiting both signs in $\Omega$ was treated in [42] for $\Omega=\mathbb{R}^{n}$ and in [6] for bounded $\Omega$. In the latter work existence of non-negative solutions (possibly with dead cores) was proved by using sub and supersolutions; some interesting uniqueness properties were also obtained. However they did not find strictly positive subsolutions and thus they were unable to prove that the obtained solutions were strictly positive (see also [9]). The existence of strictly positive solutions in bounded domains for operators in general form and $M$ changing sign has not been considered in the literature to our knowledge.

\subsection{A parabolic problem associated with (1.1)-(1.2)}

Standard linearized stability results for regular parabolic problems are readily extended to analyze the stability of the solutions of (1.1)-(1.2) that are in the positive cone of $C_{0}^{1}(\bar{\Omega})$ as steady states of the problem

$$
\begin{aligned}
& \partial u / \partial t+\mathcal{L} u=f(x, u) \quad \text { in } \Omega, \quad u=0 \quad \text { on } \partial \Omega, \\
& u(\cdot, 0)=u_{0} \quad \text { in } \Omega .
\end{aligned}
$$

In fact, if $\alpha>-1 / n$ then the operator $\mathcal{L}$ is sectorial in $L_{q}(\Omega)$ for all $q>n$ and we can apply standard results in the literature [27] to obtain a global existence result on the parabolic problem (4.24)-(4.25).

THEOREM 4.6. - In addition to the assumptions (H.1)-(H.3), let us assume that $\alpha>-1 / n$, let $u_{s} \in C^{2}(\Omega) \cap \mathcal{C}\left(=\right.$ the positive cone of $\left.C_{0}^{1}(\bar{\Omega})\right)$ be a solution of $(1.1)-$ (1.2), and let $M \equiv f_{u}\left(\cdot, u_{s}\right)$. If the principal eigenvalue of (1.4) is strictly positive (resp., strictly negative) then $u_{s}$ is an exponentially stable (resp., unstable) steady state of (4.24) in the Lyapunov sense, with the norm of $C^{1}(\bar{\Omega})$. Also, if $u_{0} \in \mathcal{C}$ then the problem (4.24)-(4.25) has a unique solution, $t \rightarrow u(\cdot, t) \in \mathcal{C}$, in a maximal existence interval, $0 \leqslant t<T \leqslant \infty$, and if $T<\infty$ then there is a sequence $\left\{t_{m}\right\}$ such that $t_{m} \nearrow T$ and either $\max \left\{u\left(x, t_{m}\right): x \in \Omega\right\} \rightarrow \infty$, or $u\left(x, t_{m}\right) \searrow 0$ for some $x \in \Omega$, or $\min \left\{\partial u\left(x, t_{m}\right) / \partial v: x \in \partial \Omega\right\} \rightarrow 0$ as $m \rightarrow \infty$.

Proof. - Let $q$ be such that $q>n$ and $1+\alpha q>0$, decompose the operator $\mathcal{L}$ as $\mathcal{L}=\mathcal{L}_{1}+\mathcal{L}_{2}$, where

$$
\mathcal{L}_{1} u \equiv \sum \frac{\partial\left(a_{i j} \partial u / \partial x_{i}\right)}{\partial x_{j}}
$$

and consider the operator $\mathcal{L}_{1}$ in $X=L_{q}(\Omega)$, with domain $D\left(\mathcal{L}_{1}\right)=W_{q}^{2}(\Omega) \cap \mathcal{C} \subset C_{0}^{1}(\bar{\Omega})$. The self-adjoint operator $\mathcal{L}_{1}$ is sectorial in $X$ (use the argument in [27, p. 32]) and if $(q+n) /(2 q)<\beta<1$ then its fractional power $\mathcal{L}_{1}^{\beta}$ is such that $\|u\|_{C^{1}(\bar{\Omega})}<K\left\|\mathcal{L}_{1}^{\beta} u\right\|_{X}$ for all $u \in D\left(\mathcal{L}_{1}\right)$ and some $K$ that is independent of $u$ [27, Theorem 1.6.1]. Also, when using assumption (H.3) and estimates (2.10), and proceeding as in Remark 2.4, the following estimates are obtained 


$$
\begin{aligned}
& \left\|f(\cdot, u)-f\left(\cdot, u_{s}\right)\right\|_{X}<K_{1}\left\|u-u_{s}\right\|_{C_{0}^{1}(\bar{\Omega})}<K_{2}\left\|\mathcal{L}_{1}^{\beta}\left(u-u_{s}\right)\right\|_{X}, \\
& \|M u\|_{X}+\left\|\mathcal{L}_{2} u\right\|_{X}<K_{3}\|u\|_{C_{0}^{1}(\bar{\Omega})}<K_{4}\left\|\mathcal{L}_{1}^{\beta} u\right\|_{X}, \\
& \left\|f(\cdot, u)-f\left(\cdot, u_{s}\right)-M\left(u-u_{s}\right)\right\|_{X}<K_{5}\left\|u-u_{s}\right\|_{C_{0}^{1}(\bar{\Omega})}^{2}<K_{6}\left\|\mathcal{L}_{1}^{\beta}\left(u-u_{s}\right)\right\|_{X}^{2},
\end{aligned}
$$

for all $u \in D\left(\mathcal{L}_{1}\right)$ and for all $u$ in a $C^{1}(\bar{\Omega})$-neighborhood of $u_{s}$ in $D\left(\mathcal{L}_{1}\right)$ respectively, with the constants $K_{1}, \ldots, K_{6}$ independent of $u$; note that (4.26)-(4.27) imply that the operator $u \rightarrow \mathcal{L}_{1} u-f(\cdot, u)$, of $X^{\beta} \equiv D\left(\mathcal{L}_{1}^{\beta}\right) \subset \mathcal{C}$ (with the norm $\|u\|_{X^{\beta}} \equiv\left\|\mathcal{L}_{1}^{\beta} u\right\|_{X}$ ) into $X$, maps bounded sets into bounded sets and is locally Lipschitzian. Then we only need to apply [27, Theorem 5.1.1] and straightforwardly modify the proofs of [27, Theorems 3.3.3 and 3.3.4] to obtain the stated results and thus to complete the proof.

Unfortunately the argument above does not apply (and, seemingly, is not straightforwardly extended) if $-1 / n \geqslant \alpha>-1$. But still, in this general case we can use the results above, in Sections 2, 3, to directly derive the following result, which should also yield the linearized stability result in Theorem 4.6 by a well-known argument $[41,43]$, provided that one has a good existence theory for the parabolic problem (4.24)-(4.25); the latter has been subsequently analyzed in [24].

THEOREM 4.7. - In addition to the assumptions (H.1)-(H.3), let $u_{s} \in C^{2}(\Omega) \cap \mathcal{C}$ (= the positive cone of $C_{0}^{1}(\bar{\Omega})$ ) be a solution of (1.1)-(1.2), and let $M \equiv f_{u}\left(\cdot, u_{s}\right)$. If the principal eigenvalue of (1.4) is strictly positive (resp., strictly negative) then there is a constant $\varepsilon_{0}>0$ and a function $U \in C^{2}(\Omega) \cap \mathcal{C}$ such that $u_{\varepsilon}=u_{s}+\varepsilon U \in \mathcal{C}$ if $|\varepsilon|<\varepsilon_{0}$, and $u_{\varepsilon}$ is a strict sub-solution (resp., super-solution) of (1.1)-(1.2) if $-\varepsilon_{0}<\varepsilon<0$, while $u_{\varepsilon}$ is a strict super-solution (resp., sub-solution) of (1.1)-(1.2) if $0<\varepsilon<\varepsilon_{0}$.

Proof. - Take two functions $u_{0}, u^{0} \in \mathcal{C}$ such that $u_{s}-u_{0}$ and $u^{0}-u_{s}$ are in $\mathcal{C}$. As in the proof of Theorem 4.1, there is a function $N \in C^{2}(\Omega)$ that satisfies (H.3') and is such that

$$
1+\left|d(x) f_{u u}(\cdot, u)\right|<N \quad \text { in } \Omega \text { if } u_{0} \leqslant u \leqslant u^{0} \text { in } \Omega .
$$

Also, according to Proposition 2.8, if the principal eigenvalue $\lambda_{0}$ of (1.4) is non-zero then the principal eigenvalue $\lambda_{1}$ of (1.5) is such that $\lambda_{1} \lambda_{0}>0$. In addition, we take an eigenfunction of (1.5) associated with $\lambda_{1}$ such that $U \in \mathcal{C}$, and the constant $\varepsilon_{0}>0$ such that $\varepsilon_{0} U<\left|\lambda_{1}\right| d(x), u_{0}<u_{-\varepsilon_{0}}$ and $u_{\varepsilon_{0}}<u^{0}$ in $\Omega$, where $u_{\varepsilon} \equiv u_{s}+\varepsilon U$ as above. Then we only need to apply the mean value theorem and take into account (4.28) to subsequently obtain

$$
\begin{aligned}
& {\left[\mathcal{L} u_{\varepsilon}-f\left(x, u_{\varepsilon}\right)-M(x) \varepsilon U\right] /\left(\lambda_{1} \varepsilon U\right)} \\
& \quad=\left\{N(x)-\left[f\left(x, u_{s}+\varepsilon U\right)-f\left(x, u_{s}\right)-M(x) \varepsilon U\right] /\left(\lambda_{1} \varepsilon U\right)\right\} \\
& \quad=N(x)-f_{\text {uи }}\left(x, u_{s}+\varepsilon \theta(x) U\right) \varepsilon U / \lambda_{1}>1 \quad \text { for all } x \in \Omega
\end{aligned}
$$

and all $\varepsilon$ such that $|\varepsilon|<\varepsilon_{0}$, where $\theta: \Omega \rightarrow \mathbb{R}$ stands for a function such that $0 \leqslant \theta \leqslant 1$ in $\Omega$. Since $\lambda_{1} \lambda_{0}>0$, the stated result follows, and the proof is complete.

As an application of Theorem 4.6 we have the following result. 
THEOREM 4.8. - Let $M$ and $\alpha_{1}$ be as in Theorem 4.4 and let $\Omega$ and $\mathcal{L}$ be as in Theorem 4.6. The parabolic problem

$$
\partial u / \partial t+\mathcal{L} u=M(x) u^{\alpha_{1}} \quad \text { in } \Omega, \quad u=0 \quad \text { on } \partial \Omega, \quad u(\cdot, 0)=u_{0} \quad \text { in } \Omega,
$$

possesses a unique solution in $0<t<\infty$ whenever $u_{0}>u_{*}$ in $\Omega$, where $u_{*} \in \mathcal{C}(=$ the positive cone of $\left.C_{0}^{1}(\bar{\Omega})\right)$ is a sub-solution of (4.13).

Proof. - As in the proof of Theorem 4.4, we can obtain a super-solution of (4.13), $u^{*}$, such that

$$
u_{*}<u_{0}=u(\cdot, 0)<u^{*} \quad \text { in } \Omega .
$$

And according to Theorem 4.6, (4.29) possesses a unique solution in a maximal existence interval $0<t<T$. And if the inequalities

$$
u_{*}<u(\cdot, t)<u^{*} \quad \text { in } \Omega
$$

hold in $0<t<t_{0}$ then they also hold at $t=t_{0}$, as readily obtained using the maximum principle in Theorem B.1 in Appendix B to the equation obtained from (4.29) when using the new variable $v=\int_{0}^{u} t^{-\alpha_{1}} d t$ and proceeding as we did to obtain (4.23). Since in addition these inequalities hold at $t=0$ we conclude that they must hold in $0<t<T$. This means that $T=\infty$ and completes the proof.

\subsection{Bifurcation problems}

If the function $f$ in the right hand side of (1.1) depends on a parameter $\lambda$ and (as in Corollary 3.2) assumptions (H.1)-(H.3) hold and $\partial^{l} f / \partial \lambda^{l}$ satisfies assumption (H.3) for all $l=1, \ldots, r$, with $r \geqslant 1$, then the results above provide the ingredients to readily analyze, quite as in the regular case, several bifurcation questions such as:

(a) The regularity of the solution branches, except at bifurcation points, via the implicit function theorem $[15,12]$.

(b) The solution branches near bifurcation points via the Crandall-Rabinowitz theorem [16] or, more generally, via the Lyapunov-Schmidt method [15,12].

(c) The global existence of solution branches via, e.g., classical results by Rabinowitz [40], based on degree theory.

For the sake of brevity we do not consider here any specific example on these applications of the results above, which actually motivated the present paper and will be considered elsewhere.

\section{Appendix A. Proof of Proposition 2.3}

The uniqueness part readily follows by a standard maximum principle. The existence part and the estimate (2.8) are obtained by regularizing the coefficients as follows. For each $\varepsilon \in] 0, \rho_{1}\left[\right.$ (with $\rho_{1}$ as defined in assumption (H.1)) we consider the problem

$$
\begin{aligned}
& -\sum a_{i j}(x) \partial^{2} u / \partial x^{i} \partial x^{j}+\varphi_{\varepsilon}(x) \sum b_{i}(x) \partial u / \partial x^{i}=\varphi_{\varepsilon}(x) M(x) v \text { in } \Omega, \\
& u=0 \text { on } \partial \Omega,
\end{aligned}
$$


where $\varphi_{\varepsilon} \in C_{0}^{1}(\bar{\Omega})$ is defined as $\varphi_{\varepsilon}(x)=\psi_{\varepsilon}(d(x))$, with

$$
\psi_{\varepsilon}(\eta)=(2 \varepsilon-\eta) \eta / \varepsilon^{2} \quad \text { if } 0<\eta<\varepsilon, \quad \psi_{\varepsilon}(\eta)=1 \quad \text { if } \eta \geqslant \varepsilon .
$$

Note that, according to assumptions (H.2), (H.3') and the estimate (2.11), $\varphi_{\varepsilon} b_{i}$ and $\varphi_{\varepsilon} M v$ are in $C^{0, \delta_{0}}(\bar{\Omega})$ if $v \in C_{0}^{1}(\bar{\Omega})$ and $\varepsilon>0$. Then (A.1) possesses a unique solution $u \in C^{2, \delta_{0}}(\bar{\Omega})$. The proof proceeds in three steps.

Step 1 . For each $\delta \in] 0, \delta_{0}[$ there are two constants, $K>0$ and $\mu \in] 0, \rho_{1}[$, independent of $v$ and $\varepsilon$, such that the solution of (A.1) satisfies

$$
\|u\|_{C^{1, \delta}\left(\bar{\Omega}_{\mu}\right)} \leqslant K\left[\|v\|_{C^{1}\left(\bar{\Omega}_{\mu}\right)}+\|u\|_{C^{0}\left(\bar{\Omega}_{\mu}\right)}+\|u\|_{C^{1, \delta}\left(\Omega \backslash \Omega_{\mu}\right)}\right],
$$

where

$$
\Omega_{\mu}=\{x \in \Omega: d(x)<\mu\} .
$$

Since $\partial \Omega$ is of class $C^{3, \gamma}$, as in [32, pp. 95-96], it can be seen that, for each $\mu<\rho_{1}$, there are two finite families of domains, $\left\{\Omega_{1 \mu}^{k}\right\}$ and $\left\{\Omega_{2 \mu}^{k}\right\}$ such that for each $k$,

(a) $\bar{\Omega}_{1 \mu}^{k} \subset \Omega_{2 \mu}^{k} \subset \bigcup_{j=1}^{m_{0}} \Omega_{1 \mu}^{k} \subset \Omega_{\mu}, \bigcup_{k} \Omega_{1 \mu}^{k}=\Omega_{\mu}$, with $m_{0}$ independent of $\mu$ and $k$.

(b) There is a $C^{2, \gamma}$-regular curvilinear, coordinate system in a neighborhood of $\bar{\Omega}_{2 \mu}^{k}$, $\xi=\xi(x)$, such that: (i) $\xi_{1}(x)=d(x)$, (ii) the domains $\Omega_{1 \mu}^{k}$ and $\Omega_{2 \mu}^{k}$ are given by

$$
\begin{aligned}
& \Omega_{i \mu}^{k}=\left\{x \in \mathbb{R}^{n}: \xi(x) \in \omega_{i \mu}^{k}\right\}, \\
& \text { with } \omega_{i \mu}^{k}=\left\{\xi \in \mathbb{R}^{n}: 0<\xi_{1}<\mu,\left|\xi_{2}\right|^{2}+\cdots+\left|\xi_{n}\right|^{2}<i \mu\right\},
\end{aligned}
$$

for $i=1$ and 2; and (iii) the $C^{2, \gamma}$-norms of the functions $\xi=\xi(x)$ and $x=x(\xi)$, in $\bar{\Omega}_{2 \mu}^{k}$ and $\bar{\omega}_{2 \mu}^{k}$ respectively, are bounded by a common constant, which is independent of $k$ and $\mu$.

Now, in the new variables, the function

$$
\left.U\left(\xi_{1}, \ldots, \xi_{n}\right)=\int_{\xi_{1}}^{\mu} u\left(y, \xi_{2}, \ldots, \xi_{n}\right)\right] d y
$$

is readily seen to satisfy

$$
\begin{aligned}
& \mathcal{L}_{0} U=\mathcal{L}_{1} U+\mathcal{L}_{2} u+\mathcal{L}_{3} u+\mathcal{L}_{4} v \quad \text { in a neighborhood of } \bar{\omega}_{2 \mu}^{k}, \\
& \partial U / \partial \xi_{1}=0 \quad \text { at } \xi_{1}=0, \quad U=0 \quad \text { at } \xi_{1}=\mu,
\end{aligned}
$$

where

$$
\begin{aligned}
& \mathcal{L}_{0} U=-\sum \tilde{a}_{i j}(\xi) \partial^{2} U / \partial \xi_{i} \partial \xi_{j}, \\
& \mathcal{L}_{1} U=\int_{\xi_{1}}^{\mu}\left[\sum\left(\partial \tilde{a}_{i j} / \partial \xi_{1}\right)\left(\partial^{2} U / \partial \xi_{i} \partial \xi_{j}\right)\right]_{\xi_{1}=y} d y, \\
& \mathcal{L}_{2} u=-\int_{\xi_{1}}^{\mu} \sum\left[\left[\tilde{b}_{i}^{1}(\xi)+\psi_{\varepsilon}\left(\xi^{1}\right) \tilde{b}_{i}^{2}(\xi)\right]\left(\partial u / \partial \xi_{i}\right)\right]_{\xi_{1}=y} d y,
\end{aligned}
$$




$$
\begin{aligned}
& \mathcal{L}_{3} u=\left[-\tilde{a}_{11} \partial u / \partial \xi_{1}+2 \sum \tilde{a}_{1 i} \partial u / \partial \xi_{i}\right]_{\xi_{1}=\mu}, \\
& \mathcal{L}_{4} v=\int_{\xi_{1}}^{\mu} \psi_{\varepsilon}(y)[\tilde{M}(\xi) v(\xi)]_{\xi_{1}=y} d y .
\end{aligned}
$$

Here $\tilde{a}_{i j}$ and $\tilde{b}_{i} \equiv \tilde{b}_{i}^{1}(\xi)+\psi_{\varepsilon}\left(\xi^{1}\right) \tilde{b}_{i}^{2}(\xi)$ denote the coefficients of the operator obtained when using the new variables in the left hand side of (A.1). Now, according to assumptions (H.2), (H.3') and property (b) above, if $0<\delta<\delta_{0}=\min \{\alpha+1, \gamma\}$ then the functions $\tilde{a}_{i j}, \xi \rightarrow \xi_{1}\left(\partial \tilde{a}_{i j} / \partial \xi_{1}\right), \tilde{b}_{i}^{1}, \xi \rightarrow \xi_{1} \tilde{b}_{i}^{2}$ and $\xi \rightarrow\left(\xi_{1}\right)^{2} \tilde{M}$ have $C^{0, \delta}\left(\bar{\omega}_{2 \mu}^{k}\right)$ norms that are uniformly bounded by a common constant, which is independent of $k$ and $\mu$. Since, in addition, $0 \leqslant \psi_{\varepsilon}\left(\xi^{1}\right) \leqslant 1$ in $0 \leqslant \xi^{1} \leqslant \mu$ (see (A.2)), we have, for all $\delta \in] 0, \delta_{0}[$,

$$
\begin{aligned}
& \left\|\mathcal{L}_{1} U\right\|_{C^{0, \delta}\left(\bar{\omega}_{2 \mu}^{k}\right)}^{(\xi)} \leqslant K \mu^{\delta_{0}-\delta}\|U\|_{C^{2, \delta}\left(\bar{\omega}_{2 \mu}^{k}\right)}^{(\xi)}, \\
& \left\|\mathcal{L}_{2} u\right\|_{C^{0, \delta}\left(\bar{\omega}_{2 \mu}^{k}\right)}^{(\xi)} \leqslant K \mu^{\delta_{0}-\delta}\|u\|_{C^{1, \delta}\left(\bar{\omega}_{2 \mu}^{k}\right)}^{(\xi)}, \\
& \left\|\mathcal{L}_{3} u\right\|_{C^{0, \delta}\left(\bar{\omega}_{2 \mu}^{k}\right)}^{(\xi)} \leqslant K\|u\|_{C^{1, \delta}\left(\Omega \backslash \Omega_{\mu}\right)}, \quad\left\|\mathcal{L}_{4} v\right\|_{C^{0, \delta}\left(\bar{\omega}_{2 \mu}^{k}\right)}^{(\xi)} \leqslant K \mu^{\delta_{0}-\delta}\|v\|_{C^{1}\left(\bar{\omega}_{2 \mu}^{k}\right)}^{(\xi)},
\end{aligned}
$$

where the constant $K$ is independent of $\varepsilon, k, \mu$ and $v$, and the superscript $(\xi)$ indicates that the new coordinates are used in the definition of the norm. The first two estimates follow straightforwardly when taking into account that $\left(\delta_{0} \equiv \min \{\gamma, \alpha+1\} \leqslant \alpha+1\right.$ and $)$

$$
\begin{aligned}
& \text { if } 0<\delta<\delta_{0} \text { and } 0<y_{1}<y_{2}<\mu \leqslant 1, \\
& \text { then } 0<y_{2}^{\alpha+1}-y_{1}^{\alpha+1} \leqslant(\alpha+1) \mu^{\alpha+1-\delta}\left|y_{2}-y_{1}\right|^{\delta} .
\end{aligned}
$$

The third estimate is a consequence of the facts that the hypersurface $\xi^{1}=\mu$ is in $\Omega \backslash \Omega_{\mu}$, and that, according to property (b) above, the Hölder norms in the variables $x$ and $\xi$ are equivalent, uniformly on $k$ and $\mu$. The last estimate is obtained when taking into account (A.14) and the inequality

$$
\begin{aligned}
& \left|v\left(\xi_{1}, \xi_{2}^{2}, \ldots, \xi_{n}^{2}\right)-v\left(\xi_{1}, \xi_{2}^{1}, \ldots, \xi_{n}^{1}\right)\right| \\
& \quad \leqslant\left|2 \xi_{1}\right|^{1-\delta}\left[\left|\xi_{2}^{2}-\xi_{2}^{1}\right|^{2}+\cdots+\left|\xi_{n}^{2}-\xi_{n}^{1}\right|^{2}\right]^{\delta / 2}\|v\|_{C^{1}\left(\bar{\omega}_{2 \mu}^{k}\right)}^{(\xi)}
\end{aligned}
$$

which holds whenever $\left(\xi_{1}, \xi_{2}^{1}, \ldots, \xi_{n}^{1}\right)$ and $\left(\xi_{1}, \xi_{2}^{2}, \ldots, \xi_{n}^{2}\right)$ are in $\omega_{2 \mu}^{k}$, and in turn is obtained when taking into account that its left hand side is bounded above by both

$$
2 \xi^{1}\|v\|_{C^{1}\left(\bar{\omega}_{2 \mu}^{k}\right)}^{(\xi)} \quad \text { and } \quad\left[\left|\xi_{2}^{2}-\xi_{2}^{1}\right|^{2}+\cdots+\left|\xi_{n}^{2}-\xi_{n}^{1}\right|^{2}\right]^{1 / 2}\|v\|_{C^{1}\left(\bar{\omega}_{2 \mu}^{k}\right)}^{(\xi)}
$$

as readily seen when applying the mean value theorem and taking into account that $v=0$ at $\xi^{1}=0$. Now, if we re-scale $\xi$ as $\xi=\mu \eta$, then in the new variables the domains $\omega_{2 \mu}^{k}$ and $\omega_{\mu}^{k}$ are fixed, and the $C^{0, \delta}$-norms of $\tilde{a}_{i j}$ are bounded above by a common constant, which is independent of $k$ and $\mu$. If we now apply a local Schauder estimate to (A.7)(A.8) in these new variables, and rewrite this estimate in terms of $\xi$, we obtain 


$$
\begin{aligned}
\|U\|_{C^{2, \delta}\left(\bar{\omega}_{\mu}^{k}\right)}^{(\xi)} \leqslant & K_{1}\left\|\mathcal{L}_{1} U+\mathcal{L}_{2} u+\mathcal{L}_{4} v\right\|_{C^{0, \delta}\left(\bar{\omega}_{2 \mu}^{k}\right)}^{(\xi)} \\
& +\mu^{-\delta} K_{2}\left\|\mathcal{L}_{3} u\right\|_{C^{0, \delta}\left(\bar{\omega}_{2 \mu}^{k}\right)}^{(\xi)}+\mu^{-(2+\delta)} K_{3}\|U\|_{C^{0}\left(\bar{\omega}_{2 \mu}^{k}\right)}^{(\xi)},
\end{aligned}
$$

where the constants $K_{1}, K_{2}$ and $K_{3}$ are independent of $\varepsilon, k, \mu$ and $v$, and we have taken into account that $\mathcal{L}_{1} U+\mathcal{L}_{2} u+\mathcal{L}_{4} v$ vanishes at $\xi^{1}=\mu$. And, when using (A.12)-(A.13) and the fact that (according to property (b) above) the Hölder-norms in the variable $\xi$ and $x$ are equivalent, uniformly in $k$ and $\mu$, we have

$$
\begin{aligned}
\|U\|_{C^{2, \delta}\left(\bar{\Omega}_{\mu}^{k}\right)} \leqslant & K_{4}\left[\mu^{\delta_{0}-\delta}\left(\|U\|_{C^{2, \delta}\left(\bar{\Omega}_{2 \mu}^{k}\right)}+\|v\|_{C^{1}\left(\bar{\Omega}_{2 \mu}^{k}\right)}\right)\right. \\
& \left.+\mu^{-\delta}\|u\|_{C^{1, \delta}\left(\Omega \backslash \Omega_{\mu}\right)}+\mu^{-1-\delta}\|u\|_{C^{0}\left(\bar{\Omega}_{2 \mu}^{k}\right)}\right],
\end{aligned}
$$

for some constant $K_{4}$ that is independent of $\varepsilon, k, \mu$ and $v$. Here we have taken into account that $U$ is independent of the curvilinear coordinate system used in $\Omega_{2 \mu}^{k}$ ( $U$ is the integral of $u$ along the normals to $\partial \Omega$, see (A.6)), and that

$$
\begin{aligned}
& \|u\|_{C^{1, \delta}\left(\bar{\Omega}_{j \mu}^{k}\right)} \leqslant\|U\|_{C^{2, \delta}\left(\bar{\Omega}_{j \mu}^{k}\right)} \text { for } j=1 \text { and } 2, \quad \text { and } \\
& \|U\|_{C^{0}\left(\bar{\Omega}_{2 \mu}^{k}\right)} \leqslant \mu\|u\|_{C^{0}\left(\bar{\Omega}_{2 \mu}^{k}\right)} .
\end{aligned}
$$

Now we choose $\mu$ such that $K_{4} \mu^{\delta_{0}-\delta} m_{0}<1 / 2$, where $m_{0}$ is as defined in property (a) above. Then, if $k_{1}$ is that value of $k$ for which the left hand side of (A.16) is greatest, we have

$$
\begin{aligned}
\|U\|_{C^{2, \delta}\left(\bar{\Omega}_{\mu}^{k_{1}}\right)} \leqslant & (1 / 2)\|U\|_{C^{2, \delta}\left(\bar{\Omega}_{\mu}^{k_{1}}\right)}+K_{4}\left[\|v\|_{C^{1}\left(\bar{\Omega}_{2 \mu}^{k_{1}}\right)}\right. \\
& \left.+\mu^{-\delta}\|u\|_{C^{1, \delta}\left(\Omega \backslash \Omega_{\mu}\right)}+\mu^{-(1+\delta)}\|u\|_{C^{0}\left(\bar{\Omega}_{2 \mu}^{k_{1}}\right)}\right],
\end{aligned}
$$

where we have used the inequality $\|U\|_{C^{2, \delta}\left(\bar{\Omega}_{2 \mu}^{k_{1}}\right)} \leqslant m_{0}\|U\|_{C^{2, \delta}\left(\bar{\Omega}_{\mu}^{k_{1}}\right)}$, which follows from property (a) above. Thus we only need to use (A.17)-(A.18) and the definition of $k_{1}$ to obtain (A.3) and complete the step.

Step 2. For each $\delta \in] 0, \delta_{0}[$ there is a constant $K$, independent of $v$ and $\varepsilon$, such that the solution of (A.1) satisfies

$$
\|u\|_{C^{1, \delta}(\bar{\Omega})} \leqslant K\left[\|v\|_{C^{1}(\bar{\Omega})}+\|u\|_{C^{0}(\bar{\Omega})}\right] .
$$

The estimate (A.19) readily follows by first selecting $\mu$ as in step 2, and then using (A.3) and the new estimate

$$
\|u\|_{C^{2, \delta}\left(\Omega \backslash \Omega_{\mu}\right)} \leqslant K_{5}\left[\|v\|_{C^{0, \delta}\left(\bar{\Omega}^{1}\right)}+\|u\|_{C^{0}\left(\bar{\Omega}^{1}\right)}\right]
$$

where $\Omega^{1}=\{x \in \Omega: d(x)>\mu / 2\}$ and $K_{5}$ is independent of $v$ and $\varepsilon$. This latter estimate is just a standard interior Schauder estimate on (A.1) (whose coefficients have $C^{1}\left(\bar{\Omega}^{1}\right)$ norms that are uniformly bounded in $0<\varepsilon<\rho_{1}$ ).

Step 3. If $v \in C_{0}^{1}(\bar{\Omega})$ then (2.7) has a solution $u \in C^{2}(\Omega) \cap C^{1, \delta}(\bar{\Omega})$ for all $\left.\delta \in\right] 0, \delta_{0}[$, and (2.8) holds with $K$ independent of $v$.

For each $m=1,2, \ldots$, let $u_{m}$ be the solution of (A.1) for $\varepsilon=\rho_{1} / m$. Let us first see that $\left\|u_{m}\right\|_{C^{0}(\bar{\Omega})}$ is bounded. To this end, we assume for contradiction that there 
is a sub-sequence, also called $\left\{u_{m}\right\}$, such that $\left\|u_{m}\right\|_{C^{0}(\bar{\Omega})} \rightarrow \infty$ as $m \rightarrow \infty$. Then $U_{m} \equiv u_{m} /\left\|u_{m}\right\|_{C^{0}(\bar{\Omega})}$ is such that, for all $m$,

$$
\begin{array}{cc}
-\sum a_{i j} \partial^{2} U_{m} / \partial x_{i} \partial x_{j}+\varphi_{\varepsilon_{m}}(x) \sum b_{i} \partial U_{m} / \partial x_{i}=\varphi_{\varepsilon_{m}}(x) M(x) v /\left\|u_{m}\right\|_{C^{0}(\bar{\Omega})} \quad \text { in } \Omega, \\
U_{m}=0 \quad \text { on } \partial \Omega, \quad \text { and }\left\|U_{m}\right\|_{C^{0}(\bar{\Omega})}=1,
\end{array}
$$

where $\varepsilon_{m} \rightarrow 0$ as $m \rightarrow \infty$. But the estimate (A.19) applied to (A.20) implies that $\left\|U_{m}\right\|_{C^{1, \delta}(\bar{\Omega})}$ is bounded if $\left.\delta \in\right] 0, \delta_{0}\left[\right.$ and, since the imbedding of $C^{1, \delta}$ into $C^{1}$ is compact, there is a subsequence, still called $\left\{u_{m}\right\}$, which converges in $C^{1}(\bar{\Omega})$ to some $U$. Now, $U \neq 0$ at some $x \in \Omega$ because $\|U\|_{C^{0}(\bar{\Omega})}=1$. Also $U \in C^{2}(\Omega)$ and satisfies

$$
\mathcal{L} U=0 \quad \text { in } \Omega, \quad U=0 \quad \text { on } \partial \Omega,
$$

as readily obtained when applying interior Schauder estimates to (A.20). But, according to standard maximum principles, (A.21) cannot have nontrivial solutions. Then a contradiction is obtained and the result follows.

Now, since $\left\|u_{m}\right\|_{C^{0}(\bar{\Omega})}$ is bounded, the estimate (A.19) readily implies that $\left\|u_{m}\right\|_{C^{1, \delta}(\bar{\Omega})}$ is also bounded for each $\delta \in] 0, \delta_{0}$ [. And, since the imbedding of $C^{1, \delta^{\prime}}$ into $C^{1, \delta}$ is compact whenever $0<\delta<\delta^{\prime}<\delta_{0}(\leqslant 1)$, for each $\left.\delta \in\right] 0, \delta_{0}[$ there is a subsequence, also called $\left\{u_{m}\right\}$, which converges in $C^{1, \delta}(\bar{\Omega})$ to some $u\left(\in C^{1, \delta}(\bar{\Omega})\right)$. Also $u \in C^{2}(\Omega)$ and satisfies (2.7) (thus the existence part of the statement follows) as readily seen when noticing that $u_{m}$ satisfies (A.1) for $\varepsilon=\varepsilon_{m}$, with $\varepsilon_{m} \rightarrow 0$ as $m \rightarrow \infty$, and applying interior Schauder estimates to this latter equation. And when applying the estimate (A.19) to this latter equation, we obtain

$$
\|u\|_{C^{1, \delta}(\bar{\Omega})} \leqslant K\left[\|v\|_{C^{1}(\bar{\Omega})}+\|u\|_{C^{0}(\bar{\Omega})}\right]
$$

where $K$ is independent of $v$.

Finally, $u$ and $v$ satisfy (2.8), which follows from (A.22) and the estimate

$$
\|u\|_{C^{0}(\bar{\Omega})} \leqslant K\|v\|_{C^{1}(\bar{\Omega})},
$$

with $K$ independent of $v$. And this latter estimate is readily obtained from (A.22) by a standard contradiction argument, alike to the one already used above (if (A.23) does not hold, then there is a sequence $\left\{v_{m}\right\} \subset C_{0}^{1}(\bar{\Omega})$ such that $\left\|v_{m}\right\|_{C^{1}(\bar{\Omega})}=1$ for all $m$, and the corresponding solutions of (2.7) are such that $\left\|u_{m}\right\|_{C^{0}(\bar{\Omega})} \rightarrow \infty$ as $m \rightarrow \infty$; but then $U_{m}=u_{m} /\left\|u_{m}\right\|_{C^{0}(\bar{\Omega})}$ possesses a subsequence that converges in $C^{2}(\Omega) \cap C^{1}(\bar{\Omega})$ to a nontrivial solution of (A.21), which cannot exist). This completes the step, and the proof of Proposition 2.2.

\section{Appendix B. A strong maximum principle for second order equations with locally bounded coefficients}

Here we derive a strong maximum principle for some elliptic and parabolic inequalities with locally bounded coefficients, such as those appearing in this paper. The elliptic case was already considered in [39,30], under essentially the same assumptions 
made below, but we have been unable to find a proof for the parabolic case in the literature. For the sake of brevity we first consider the parabolic case, which contains the elliptic one as a particular case. Of course, the elliptic case could have been directly treated in a similar way.

THEOREM B.1. - Let $u \in C^{1}\left(\bar{\Omega} \times\left[t_{0}, t_{1}\right]\right)$ be such that $u(\cdot, t) \in C^{2}(\Omega) \cap C^{1, \delta}(\bar{\Omega})$ for all $t \in\left[t_{0}, t_{1}\right]$ and

$$
N(x) \partial u / \partial t+\mathcal{L} u+M(x) u \leqslant 0 \quad \text { in } \Omega \times] t_{0}, t_{1}[,
$$

where $0<\delta<1, \Omega, \mathcal{L}, M$ and $N$ satisfy assumptions (H.1)-(H.2), (H.3') and (H.4), and $M \geqslant 0$ in $\Omega$. Let us assume also that $u \leqslant 0$ in $\Omega \times] t_{0}, t_{1}\left[\right.$, and that $u\left(x_{0}, t_{1}\right)=0$. Then the following properties hold:

(i) If $x_{0} \in \Omega$ then $u=0$ in $\bar{\Omega} \times\left[t_{0}, t_{1}\right]$.

(ii) If $x_{0} \in \partial \Omega$ and $u<0$ in $\left.\Omega \times\right] t_{0}, t_{1}\left[\right.$ then $\partial u / \partial v>0$ at $\left(x_{0}, t_{1}\right)$.

Proof. - Since the coefficients of the linear operator in the left hand side of (B.1) are locally bounded in $\Omega$ and $N>0$ in $\Omega$, property (i) readily follows when applying the standard strong maximum principle [38].

In order to prove property (ii) assume for contradiction that

$$
u<0 \quad \text { in } \Omega \times] t_{0}, t_{1}\left[\text { and } u=\partial u / \partial v=0 \text { at }\left(x_{0}, t_{1}\right) .\right.
$$

Since, in addition, $u\left(\cdot, t_{1}\right) \in C^{1, \delta}\left(\bar{\Omega}\right.$ there is a constant $k_{2}>0$ such that

$$
\left|u\left(x, t_{1}\right)\right|=\left|u\left(x, t_{1}\right)-u\left(x_{0}, t_{1}\right)\right| \leqslant k_{2}\left|x-x_{0}\right|^{1+\delta} \quad \text { for all } x \in \Omega .
$$

On the other hand, $\Omega$ satisfies the interior sphere condition (because of assumption (H.1)), i.e., there is a hypersphere $H$, with center at $y_{0} \in \Omega$ and radius $\rho_{1}>0$ such that $H \subset \Omega \cup \partial \Omega$ and $H \cap \partial \Omega=\left\{x_{0}\right\}$. Let us consider the function

$$
v(x, t)=\left[t-t_{1}+\rho_{1}-\rho(x)\right]^{1+\delta / 2}, \quad \text { with } \rho(x)=\left|x-y_{0}\right|,
$$

which (when proceeding as in the proof of Lemma 2.1) is seen to satisfy

$$
\begin{aligned}
& N \partial v / \partial t+\mathcal{L} v+M v<0 \quad \text { in } \\
& \left.\left.A=\{(x, t) \in \Omega \times] t_{0}, t_{1}\right]: \rho(x)>\rho_{2}, \rho_{1}-\rho(x)>t_{1}-t \geqslant 0\right\},
\end{aligned}
$$

provided that $\rho_{2}$ is appropriately close to $\rho_{1}$. In that case the function $w_{\varepsilon} \equiv u+\varepsilon v$ is such that (see (B.1)) $N(x) \partial w_{\varepsilon} / \partial t+\mathcal{L} w_{\varepsilon}+M(x) w_{\varepsilon}<0$ in $A$, whenever $\varepsilon>0$; thus the standard maximum principle [38] implies that the maximum of $w_{\varepsilon}$ in $A$ can be attained neither at an interior point of $A$ nor at $t=t_{1}$. Thus this maximum must be attained either at $\rho_{1}-\rho(x)=t_{1}-t$ or at $\rho(x)=\rho_{2}$; but (a) $w_{\varepsilon} \equiv u+\varepsilon v=u \leqslant 0$ if $\rho_{1}-\rho(x)=t_{1}-t \geqslant$ 0 and $\varepsilon>0$, and (b) $w_{\varepsilon} \equiv u+\varepsilon v<0$ if $\rho(x)=\rho_{2}, \rho_{1}-\rho_{2} \geqslant t_{1}-t \geqslant 0$ and $\varepsilon>0$ is appropriately small (see (B.2) and (B.4)). Thus for that value of $\varepsilon, w \leqslant 0$ (i.e., $u \leqslant-\varepsilon v$ ) in $A$. This property holds, in particular, on the rectilinear segment $S$ of $\Omega \times\left\{t_{1}\right\}$ joining $\left(y_{0}, t_{1}\right)$ and $\left(x_{0}, t_{1}\right)$, where $\rho_{1}-\rho(x)=d(x)$. Then we have

$$
u\left(x, t_{1}\right) \leqslant-\varepsilon d(x)^{1+\delta / 2} \quad \text { in } x \in S \cap A \subset \Omega \quad \text { (i.e., if } d(x)>0 \text { is sufficiently small). }
$$


Since $\varepsilon>0$ and $\delta>0$, this inequality is in contradiction with (B.3), and the proof is complete.

The elliptic case is reduced to the parabolic one as usual, just by noticing that if a function $u=u(x)$ satisfies the elliptic inequality (B.6) below then it also satisfies (B.1), and if that function attains a maximum at $x_{0} \in \bar{\Omega}$, then it also attains the maximum at $\left(x_{0}, t\right) \in \bar{\Omega} \times \mathbb{R}$ for all $t$. Thus the following result follows.

THEOREM B.2. - Let $u \in C^{2}(\Omega) \cap C^{1, \delta}(\bar{\Omega})$ be such that

$$
\mathcal{L} u+M(x) u \leqslant 0 \quad \text { a.e. in } \Omega,
$$

where $\delta, \Omega, \mathcal{L}$ and $M$ are as in Theorem B.1. Let us assume that $u \leqslant 0$ in $\bar{\Omega}$ and $u\left(x_{0}\right)=0$ for some $x_{0} \in \bar{\Omega}$. Then the following properties hold:

(i) If $x_{0} \in \Omega$ then $u=0$ in $\bar{\Omega}$.

(ii) If $x_{0} \in \partial \Omega$ and $u<0$ in $\Omega$ then $\partial u / \partial v>0$ at $x_{0}$.

\section{REFERENCES}

[1] R.A. Adams, Sobolev Spaces, Academic Press, 1975.

[2] S. Agmon, A. Douglis, L. Nirenberg, Estimates near the boundary for solutions of elliptic partial differential equations satisfying general boundary conditions I, Comm. Pure Appl. Math. 12 (1959) 623-727.

[3] H. Amann, Fixed point equations and nonlinear eigenvalue problems in ordered Banach spaces, SIAM Rev. 18 (1976) 620-709.

[4] A. Ambrosetti, H. Brezis, G. Cerami, Combined effects of concave and convex nonlinearities in some elliptic problems, J. Funct. Anal. 122 (1994) 519-543.

[5] D. Aronson, M.G. Crandall, L.A. Peletier, Stabilization of solutions of a degenerate nonlinear diffusion problem, Nonlinear Analysis TMA 16 (1982) 1001-1022.

[6] C. Bandle, M.A. Pozio, A. Tesei, The asymptotic behavior of the solutions of degenerate parabolic equations, Trans. Amer. Math. Soc. 303 (1987) 487-501.

[7] H. Berestycki, L. Nirenberg, S.R.S. Varadhan, The principal eigenvalue and maximum principle for second-order elliptic operators in general domains, Comm. Pure Appl. Math. 47 (1994) 47-92.

[8] M. Bertsch, R. Rostamian, The principle of linearized stability for a class of degenerate diffusion equations, J. Differential Equations 57 (1985) 373-405.

[9] L. Boccardo, L. Orsina, Sublinear elliptic equations in $L^{1}$, Houston Math. J. 20 (1994) 99114.

[10] H. Brezis, S. Kamin, Sublinear elliptic equations in $\mathbf{R}^{n}$, Manuscripta Math. 74 (1992) $87-$ 106.

[11] H. Brezis, L. Oswald, Remarks on sublinear elliptic equations, Nonlinear Analysis TMA 10 (1986) 55-64.

[12] S.N. Chow, J.K. Hale, Methods of Bifurcation Theory, Springer-Verlag, 1982.

[13] Ph. Clément, D.G. de Figueiredo, E. Mitidieri, A priori estimates for positive solutions of semilinear elliptic systems via Hardy-Sobolev inequalities, Pitman Research Notes 343 (1996) 73-91.

[14] D. Cohen, T. Laetsch, Nonlinear boundary value problems suggested by chemical reactor theory, J. Differential Equations 7 (1970) 217-226. 
[15] M.G. Crandall, An introduction to constructive aspects of bifurcation and the implicit function theorem, in: P.H. Rabinowitz (Ed.), Applications of Bifurcation Theory, Academic Press, New York, 1977, pp. 1-35.

[16] M.G. Crandall, P.H. Rabinowitz, Bifurcation from simple eigenvalues, J. Funct. Anal. 8 (1971) 321-340.

[17] M.G. Crandall, P.H. Rabinowitz, Bifurcation, perturbation of simple eigenvalues, and linearized stability, Arch. Rat. Mech. Anal. 52 (1973) 161-180.

[18] M.G. Crandall, P.H. Rabinowitz, L. Tartar, On a Dirichlet problem with a singular nonlinearity, Comm. Partial Differential Equations 2 (1977) 193-222.

[19] R. Dautray, J.L. Lions, Mathematical Analysis and Numerical Methods for Science and Technology, Vol. 3, Springer-Verlag, Berlin, 1990.

[20] J.I. Díaz, Nonlinear Partial Differential Equations and Free Boundaries, Pitman, Boston, 1985.

[21] J. Dieudonné, Foundations of Modern Analysis, Academic Press, New York, 1960.

[22] M. Donsker, S.R.S. Varadhan, On the principal eigenvalue of second-order elliptic differential operators, Comm. Pure Appl. Math. 29 (1976) 595-621.

[23] C. Faber, Beweis das unter allen homogenen membranen von gleicher fläche und gleicher spannung die kreisförmige den tiefsten grundton gibt, Sitzunsber, Bayer. Akad. der Wiss. Math. Phys. (1923) 169-172.

[24] W. Fulks, J.S. Maybee, A singular nonlinear equation, Osaka J. Math. 12 (1960) 1-19.

[25] W.S.C. Gurney, R.N. Nisbet, The regulation of inhomogeneous populations, J. Theor. Biol. 52 (1975) 441-457.

[26] M.E. Gurtin, R.C. MacCamy, On the diffusion of biological populations, Math. Biosci. 33 (1977) 35-49.

[27] D. Henry, Geometric Theory of Parabolic Equations, in: Lecture Notes in Math., Vol. 840, Springer-Verlag, Berlin, 1981.

[28] J. Hernández, in preparation, 1999.

[29] P. Hess, T. Kato, On some linear and nonlinear eigenvalue problems with an indefinite weight function, Comm. Partial Differential Equations 5 (1980) 999-1030.

[30] L.I. Kamynin, B.N. Khimchenko, Development of Aleksandrov's theory of the isotropic strict extremum principle, Differential Equations (English translation) 16 (1980) 181-189.

[31] E. Krahn, Über eine von Rayleigh formulierte minimaleigenschaft des kreises, Math. Ann. 91 (1925) 97-100.

[32] O.A. Ladyženskaja, V.A. Solonnikov, N.N. Ural'ceva, Linear and Quasilinear Equations of Parabolic Type, American Mathematical Society, Providence, 1968.

[33] T. Laetsch, Uniqueness of sublinear boundary value problems, J. Differential Equations 13 (1973) 13-23.

[34] J. López-Gómez, The maximum principle and the existence of principal eigenvalues for some linear weighted boundary value problems, J. Differential Equations 127 (1996) 263 294.

[35] A. Manes, A.M. Micheletti, Un estensione della teoria variazonale classica degli autovalori per operatori ellittici del secondo ordine, Boll. Un. Mat. Ital. 7 (1973) 285-301.

[36] T. Namba, Density-dependent dispersal and spatial distribution of a population, J. Theor. Biol. 86 (1980) 351-363.

[37] C.V. Pao, Nonlinear Parabolic and Elliptic Equations, Plenum Press, New York, 1992.

[38] M.H. Protter, H.F. Weinberger, Maximum Principles in Differential Equations, SpringerVerlag, Berlin, 1984. 
[39] C. Pucci, Propietà di massimo e minimo delle soluzioni di equazioni a derivate parziali del secondo ordine di tipo ellittico e parabolico, Atti. Acad. Naz. Lincei Rend. Cl. Sci. Fis. Mat. 23 (1957) 370-375.

[40] P.H. Rabinowitz, Théorie du degré topologique et applications à des problèmes aux limites non linéaires, in: Lecture Notes, Lab. Analyse Numérique, Univ. Paris VI, Paris, 1975.

[41] D.H. Sattinger, Monotone methods in nonlinear elliptic and parabolic boundary value problems, Indiana Univ. Math. J. 21 (1972) 979-1000.

[42] M. Schatzman, Stationary solutions and asymptotic behavior of a quasilinear degenerate parabolic equation, Indiana Univ. Math. J. 33 (1984) 1-29.

[43] J. Smoller, Shock Waves and Reaction-Diffusion Equations, Springer-Verlag, Berlin, 1983.

[44] J. Spruck, Uniqueness of a diffusion model of population biology, Comm. Partial Differential Equations 8 (1983) 1605-1620. 